\title{
On Some Properties of Dyadic Operators
}

\author{
Heng Gu, Qingying Xue* and Kôzô Yabuta
}

\begin{abstract}
In this paper, the objects of our investigation are some dyadic operators, including dyadic shifts, multilinear paraproducts and multilinear Haar multipliers. We mainly focus on the continuity and compactness of these operators. First, we consider the continuity properties of these operators. Then, by the Fréchet-KolmogorovRiesz-Tsuji theorem, the non-compactness properties of these dyadic operators will be studied. Moreover, we show that their commutators are compact with CMO functions, which is quite different from the non-compactness properties of these dyadic operators. These results are similar to those for Calderón-Zygmund singular integral operators.
\end{abstract}

\section{Introduction}

It is well known that the dyadic operators, such as paraproducts, Haar multipliers and dyadic shifts, play very important roles in Harmonic Analysis. The study of paraproducts may be traced back to the famous work of Bony in [2]. Since then, many works had been done in this field. Among those achievements is the celebrated work of David and Journé [3]. Using the techniques of paraproducts, David and Journé established the $T(1)$ theorem and thus gave a boundedness criterion for generalized Calderón-Zygmund operators. The investigation of Haar multipliers may be dated back to the $A_{2}$ conjecture for Haar multipliers consider by Wittwer in [18]. Subsequently, using the combination of Bellman function technique and heat extension, Petermichl and Volberg extended the same result to Beurling-Ahlfors transforms in [14]. As for the dyadic shifts, it is known that an elementary dyadic shift with parameter $(m, n)(m, n \in \mathbb{N})$ is an operator given by

$$
\begin{aligned}
& \mathbb{S} f(x)=\sum_{I \in \mathcal{D}} \frac{1}{|I|} \int_{I} a_{I}(x, y) f(y) d y=\sum_{I \in \mathcal{D}} \sum_{I^{\prime}, I^{\prime \prime} \in \mathcal{D}, I^{\prime}, I^{\prime \prime} \subset I} \frac{1}{|I|}\left\langle f, h_{I^{\prime}}\right\rangle h_{I^{\prime \prime}} \\
& l\left(I^{\prime}\right)=2^{-m} l(I) \\
& l\left(I^{\prime \prime}\right)=2^{-n} l(I)
\end{aligned}
$$

Received May 7, 2021; Accepted November 15, 2021.

Communicated by Sanghyuk Lee.

2020 Mathematics Subject Classification. 42B20, 47G10.

Key words and phrases. paraproducts, dyadic shifts, Haar multipliers, commutators of Haar multipliers, continuity, compactness.

The authors were supported partly by NSFC (No. 11871101), 111 Project and the National Key Research and Development Program of China (Grant No. 2020YFA0712900). The third author was supported partly by Grant-in-Aid for Scientific Research (C) Nr. 15K04942, Japan Society for the Promotion of Science.

*Corresponding author. 
where $h_{I^{\prime}}$ and $h_{I^{\prime \prime}}$ are Haar functions for the cubes $I^{\prime}$ and $I^{\prime \prime}$ respectively in $\mathbb{R}^{d}$, subject to normalization $\left\|h_{I^{\prime}}\right\|_{\infty} \cdot\left\|h_{I^{\prime \prime}}\right\|_{\infty} \leq 1$ and

$$
a_{I}=\sum_{\substack{I^{\prime}, I^{\prime \prime} \in \mathcal{D}, I^{\prime}, I^{\prime \prime} \subset I \\ l\left(I^{\prime}\right)=2^{-m} l(I), l\left(I^{\prime \prime}\right)=2^{-n} l(I)}} h_{I^{\prime}}(y) h_{I^{\prime \prime}}(x) .
$$

The number $r=\max (m, n)$ is called the complexity of the dyadic shift. There are two important works in the earlier stage of investigation. The first one is given in 12 which concerned with the boundedness of dyadic shifts. The second one is given by Lacey, Petermichl and Reguera [10] which demonstrates the $A_{2}$ conjecture for general dyadic shifts. A recent nice work [6] states that an arbitrary Calderón-Zygmund operator can be presented as an average of random dyadic shifts and random dyadic paraproducts. This demonstrates the importance of the dyadic shifts and people are beginning to pay more attention to these operators.

Still more recently, the following multilinear dyadic paraproducts $\pi_{b}^{\vec{\alpha}}$, Haar multipliers $P^{\vec{\alpha}}$ and $T_{\epsilon}^{\vec{\alpha}}$ have been introduced and studied by Kunwar [9]:

$$
\begin{gathered}
\pi_{b}^{\vec{\alpha}}(\vec{f})(x)=\sum_{I \in D} \frac{\left\langle b, h_{I}\right\rangle}{|I|}\left(\prod_{j=1}^{m} \frac{\left\langle f_{j}, h_{I}^{1+\alpha_{j}}\right\rangle}{|I|}\right) h_{I}^{1+\sigma(\vec{\alpha})}, \quad \vec{\alpha}=\left(\alpha_{1}, \ldots, \alpha_{m}\right) \in\{0,1\}^{m}, \\
P^{\vec{\alpha}}(\vec{f})(x)=\sum_{I \in D}\left(\prod_{j=1}^{m} \frac{\left\langle f_{j}, h_{I}^{1+\alpha_{j}}\right\rangle}{|I|}\right) h_{I}^{\sigma(\vec{\alpha})}, \quad \vec{\alpha} \in\{0,1\}^{m} \backslash\{1, \ldots, 1\}, \\
T_{\epsilon}^{\vec{\alpha}}(\vec{f})(x)=\sum_{I \in \mathcal{D}} \epsilon_{I}\left(\prod_{j=1}^{m} \frac{\left\langle f_{j}, h_{I}^{1+\alpha_{j}}\right\rangle}{|I|}\right) h_{I}^{\sigma(\vec{\alpha})}, \quad \vec{\alpha} \in\{0,1\}^{m} \backslash\{1, \ldots, 1\},
\end{gathered}
$$

where $b \in \mathrm{BMO}^{d}$, and $\epsilon=\left\{\epsilon_{I}\right\}_{I \in \mathcal{D}}$ is bounded and $\sigma(\vec{\alpha})$ is denoted to be the number of 0 components in $\vec{\alpha}$.

In [9], Kunwar investigated the strong and weak type boundedness properties of $\pi_{b}^{\vec{\alpha}}$ and its commutators. Moreover, Kunwar 9] demonstrated that

$$
f_{1} \cdots f_{m}=\sum_{\vec{\alpha} \in\{0,1\}^{m} \backslash\{(1, \ldots, 1)\}} P^{\vec{\alpha}}(\vec{f}) \quad \text { for } f_{j} \in L^{p_{j}}(\mathbb{R}) .
$$

If $1<p_{1}, \ldots, p_{m}<\infty$ with $\frac{1}{p}=\sum_{i=1}^{m} \frac{1}{p_{i}}$ and $b \in \mathrm{BMO}^{d}$, Kunwar 8 showed that the Haar multipliers and their commutators enjoy the properties that

$$
T_{\epsilon}^{\vec{\alpha}}: L^{p_{1}} \times L^{p_{2}} \times \cdots \times L^{p_{m}} \rightarrow L^{p}
$$

and

$$
\left[b, T_{\epsilon}^{\vec{\alpha}}\right]_{j}: L^{p_{1}} \times L^{p_{2}} \times \cdots \times L^{p_{m}} \rightarrow L^{p} \quad \text { for } j=1, \ldots, m,
$$

where $\left[b, T_{\epsilon}^{\vec{\alpha}}\right]_{j}$ is denoted to be the commutator of $T_{\epsilon}^{\vec{\alpha}}$ in the $j$-th entry. 
This paper will be devoted to investigate the continuity and compactness of the above dyadic type operators, including their commutators. First, we consider the continuity properties of them and get the following result.

Theorem 1.1 (Continuity of dyadic operators). The following statements hold:

(i) Let $|\nabla f| \in L^{\infty}\left(\mathbb{R}^{d}\right)$. Then $\mathbb{S} f(x)$ is almost everywhere continuous.

(ii) Let $\vec{\alpha} \in\{0,1\}^{m} \backslash\{(1, \ldots, 1)\}$ and $\epsilon=\left\{\epsilon_{I}\right\}$ be a bounded sequence. Suppose that $f_{j}^{\prime}$ is bounded when $\alpha_{j}=0$ and $f_{j}$ is bounded when $\alpha_{j}=1$ in $\mathbb{R}$. Then $\pi_{b}^{\vec{\alpha}}(\vec{f})(x)$ and $T_{\epsilon}^{\vec{\alpha}}(\vec{f})(x)$ are almost everywhere continuous.

Remark 1.2. For dyadic paraproducts $\pi_{b}^{\vec{\alpha}}(\vec{f})(x)$, when $\vec{\alpha}=\{(1, \ldots, 1)\}$, then $\pi_{b}^{\vec{\alpha}}(\vec{f})(x)$ is also almost everywhere continuous if $b^{\prime}(x)$ is bounded and for all $f_{j}$ is bounded in $\mathbb{R}$. The square of the Littlewood-Paley square function $S f(x)=\left(\sum_{I \in \mathcal{D}}\left(\frac{\left\langle f, h_{I}\right\rangle}{|I|}\right)^{2} \chi_{I}\right)^{1 / 2}$ and Haar multipliers $P^{\vec{\alpha}}(\vec{f})(x)$ are special cases of $T_{\epsilon}^{\vec{\alpha}}(\vec{f})(x)$. Therefore, they are also almost everywhere continuous.

There are many results about the compactness of the non-dyadic operators. For example, [16, 17 are some nice works in the earlier stage. Recently, the authors in [1, 4 studied the compactness of bilinear operators and their commutators. But there is no compactness or non-compactness results for dyadic operators. Thus, it is quite natural to ask whether these dyadic operators are compact or not. Below, we will give a negative answer to this question.

Theorem 1.3 (Noncompactness of dyadic operators). (i) Let $\epsilon=\left\{\epsilon_{I}\right\}$ be a bounded sequence and suppose that there exists a constant $A>0$ such that $\#\left\{I \in \mathcal{D}:\left|\epsilon_{I}\right| \geq\right.$ $A\}=\infty$. Let $\frac{1}{p}=\frac{1}{p_{1}}+\cdots+\frac{1}{p_{m}}$ with $1<p_{1}, \ldots, p_{m}<\infty$. Then $T_{\epsilon}^{\vec{\alpha}}$ is not a compact operator from $L^{p_{1}}(\mathbb{R}) \times \cdots \times L^{p_{m}}(\mathbb{R})$ to $L^{p}(\mathbb{R})$ for $\vec{\alpha} \in\{0,1\}^{m} \backslash\{(1, \ldots, 1)\}$.

(ii) Let $m, n \in \mathbb{N}$ and suppose that there exists a constant $A>0$ such that

$$
\begin{gathered}
\#\left\{I \in \mathcal{D}: A \leq\left\|h_{I^{\prime}}\right\|_{\infty} \cdot\left\|h_{I^{\prime \prime}}\right\|_{\infty} \leq 1 \text { for some } I^{\prime}, I^{\prime \prime} \in \mathcal{D}, I^{\prime}, I^{\prime \prime} \subset I,\right. \\
\left.l\left(I^{\prime}\right)=2^{-m} l(I), l\left(I^{\prime \prime}\right)=2^{-n} l(I)\right\}=\infty
\end{gathered}
$$

Then, dyadic shift with parameters $(m, n)$ is not a compact operator.

There also exists $b \in L^{\infty} \subset \mathrm{BMO}$ such that $\pi_{b}^{\vec{\alpha}}$ is not a compact operator. However, for $b \in \mathrm{CMO}$, it can be shown that $\pi_{b}^{\vec{\alpha}}$ is a compact operator. Consequently, we get

Theorem 1.4 (Compactness of $\pi_{b}^{\vec{\alpha}}$ ). Let $b \in \mathrm{CMO}$ and $\frac{1}{p}=\frac{1}{p_{1}}+\cdots+\frac{1}{p_{m}}$ with $1<$ $p_{1}, \ldots, p_{m}<\infty$. Then $\pi_{b}^{\vec{\alpha}}$ is a compact operator from $L^{p_{1}}(\mathbb{R}) \times \cdots \times L^{p_{m}}(\mathbb{R})$ to $L^{p}(\mathbb{R})$ for $\vec{\alpha} \in\{0,1\}^{m}$. 
Nevertheless, like in [1,4] for many non-dyadic operators, they may be not compact but their commutators and iterated commutators can be compact. Therefore, we try to figure out whether the commutators and the iterated commutators of these dyadic operators are compact or not. First, following the usual definition of commutators $[b, T](f)=$ $b T(f)-T(b f)$, we define the iterated commutators of Haar multipliers $T_{\epsilon, \Pi \mathbf{b}}^{\vec{\alpha}}$ by

$$
T_{\epsilon, \Pi \mathbf{b}}^{\vec{\alpha}}(\vec{f})=\left[b_{1},\left[b_{2}, \ldots\left[b_{m-1}\left[b_{m}, T_{\epsilon}^{\vec{\alpha}}\right]_{m}\right]_{m-1} \ldots\right]_{2}\right]_{1}(\vec{f}) .
$$

We formulate the results for the compactness of the commutators as follows:

Theorem 1.5 (Compactness of commutators). Let $\epsilon=\left\{\epsilon_{I}\right\}$ be a bounded sequence and $\frac{1}{p}=\frac{1}{p_{1}}+\cdots+\frac{1}{p_{m}}$ with $1<p_{1}, \ldots, p_{m}<\infty$. The following statements hold:

(i) Let $b \in \mathrm{CMO}$. Then $\left[b, T_{\epsilon}^{\vec{\alpha}}\right]_{i}$ is a compact operator from $L^{p_{1}}(\mathbb{R}) \times \cdots \times L^{p_{m}}(\mathbb{R})$ to $L^{p}(\mathbb{R})$ for all $\vec{\alpha} \in\{0,1\}^{m} \backslash\{(1, \ldots, 1)\}$ and $1 \leq i \leq m$.

(ii) Let $\vec{b}=\left(b_{1}, \ldots, b_{m}\right) \in \mathrm{CMO}^{m}$. Then $T_{\epsilon, \Pi \mathbf{b}}^{\vec{\alpha}}$ is a compact operator from $L^{p_{1}}(\mathbb{R}) \times$ $\cdots \times L^{p_{m}}(\mathbb{R})$ to $L^{p}(\mathbb{R})$ for $\vec{\alpha} \in\{0,1\}^{m} \backslash\{1, \ldots, 1\}$.

(iii) Let $b \in \mathrm{CMO}$. Then $[b, \mathbb{S}]$ is a compact operator from $L^{p}\left(\mathbb{R}^{d}\right)$ to $L^{p}\left(\mathbb{R}^{d}\right)$.

The rest of this article is organized as follows. Some preliminaries which will be used later are given in Section 2. The proof of Theorem 1.1 will be given in Section 3. Section 4 will be devoted to demonstrate Theorems 1.3 and 1.4 . The proof of Theorem 1.5 will be presented in Section 5

\section{Preliminaries}

\subsection{Standard dyadic lattices and Haar system}

The standard dyadic system in $\mathbb{R}^{d}$ is

$$
\mathcal{D}:=\bigcup_{k \in \mathbb{Z}} \mathcal{D}_{k}, \quad \mathcal{D}_{k}:=\left\{2^{k}\left([0,1)^{d}+m\right): m \in \mathbb{Z}^{d}\right\} .
$$

For $I \in \mathcal{D}, I^{(j)}$ is denoted to be the $j$-th dyadic ancestor of $I\left(2^{j} l(I)=l\left(I^{(j)}\right)\right.$ and $\left.I \subset I^{(j)}\right)$, $j \in \mathbb{Z}_{+} \cup\{0\}$. Given a cube $I=x+[0,1)^{d}$, let $\operatorname{ch}(I):=\left\{x+\eta l / 2+[0,1 / 2)^{d}: \eta \in\{0,1\}^{d}\right\}$ be the collection of dyadic children of $I$. Thus $\mathcal{D}_{k-1}=\bigcup\left\{\operatorname{ch}(I): I \in \mathcal{D}_{k}\right\}$. Associated to the dyadic cube $I$ there is a Haar function $h_{I}$ which is defined by

$$
h_{I}=\sum_{J \in\{\operatorname{ch}(I)\}} \alpha_{J} 1_{J}, \quad \sum_{J \in\{\operatorname{ch}(I)\}} \alpha_{J}|J|=0 .
$$

When $I$ is a dyadic interval and let $I_{+}$and $I_{-}$be the right and left halves of $I$, then, the Haar function $h_{I}$ is defined by $h_{I}=1_{I_{+}}-1_{I_{-}}$. It is well known that the collection of all Haar functions $\left\{\frac{h_{I}}{\sqrt{|I|}}: I \in \mathcal{D}\right\}$ is an orthonormal basis of $L^{2}(\mathbb{R})$ and an unconditional basis of $L^{p}(\mathbb{R})$ for $1<p<\infty$. 


\subsection{Multilinear weights}

Following the notation in [11], for $m$ exponents $p_{1}, \ldots, p_{m}$, we write $p$ for the number given by $1 / p=1 / p_{1}+\cdots+1 / p_{m}$ and $\vec{P}$ for the vector $\vec{P}=\left(p_{1}, \ldots, p_{m}\right)$.

Definition 2.1 (Multiple weights, 11$]$ ). For $1 \leq p_{1}, \ldots, p_{m}<\infty$ and a multiple weight $\vec{\omega}=\left(\omega_{1}, \ldots, \omega_{m}\right)$, we say that $\vec{\omega}$ satisfies the multilinear $A_{\vec{P}}$ condition if

$$
\sup _{I}\left(\frac{1}{|I|} \int_{I} \nu_{\vec{\omega}}\right)^{1 / p} \prod_{j=1}^{m}\left(\frac{1}{|I|} \int_{I} \omega_{j}^{1-p_{j}^{\prime}}\right)^{1 / p_{j}}<\infty
$$

where $\nu_{\vec{\omega}}=\prod_{j=1}^{m} \omega_{j}^{p / p_{j}}$. When $p_{j}=1,\left(\frac{1}{|I|} \int_{I} \omega_{j}^{1-p_{j}^{\prime}}\right)^{1 / p_{j}}$ is understood as $\left\|\omega_{j}^{-1}\right\|_{L^{\infty}(I)}$.

By Hölder's inequality, it is easy to see that

$$
\prod_{j=1}^{m} A_{P_{j}} \subset A_{\vec{P}}
$$

Moreover, if $\vec{\omega} \in A_{\vec{P}}$, then we have $\nu_{\vec{\omega}} \in A_{m p}$. We will similarly denote the dyadic multilinear $A_{\vec{P}}$ class by $A_{\vec{P}}^{d}$.

\subsection{BMO space}

For a locally integrable function $b$ on $\mathbb{R}$, set

$$
\|b\|_{\mathrm{BMO}}=\sup _{I} \frac{1}{|I|} \int_{I}\left|b(x)-\langle b\rangle_{I}\right| d x
$$

where the supremum is taken over all intervals $I$ in $\mathbb{R}$, and $\langle b\rangle_{I}=|I|^{-1} \int_{I} b(x) d x$. The function $b$ is called of bounded mean oscillation if $\|b\|_{\mathrm{BMO}}<\infty$ and $\mathrm{BMO}(\mathbb{R})$ is the set of all locally integrable functions $b$ on $\mathbb{R}$ with $\|b\|_{\text {BMO }}<\infty$. We define CMO to be the closure of $C_{c}^{\infty}$ in the BMO norm.

If we take the supremum over all dyadic intervals in $\mathbb{R}$, we get a larger space of dyadic $\mathrm{BMO}$ functions which is denoted by $\mathrm{BMO}^{d}$. For $1<r<\infty$, define

$$
\mathrm{BMO}_{r}=\left\{b \in L_{\mathrm{loc}}^{p}:\|b\|_{\mathrm{BMO}_{r}}<\infty\right\}
$$

where $\|b\|_{\mathrm{BMO}_{r}}:=\left(\sup _{I} \frac{1}{|I|} \int_{I}\left|b(x)-\langle b\rangle_{I}\right|^{r} d x\right)^{\frac{1}{r}}$. For any $1<r<\infty$, the norms $\|b\|_{\mathrm{BMO}_{r}}$ and $\|b\|_{\mathrm{BMO}}$ are equivalent (see [5,7]). For $r=2$, it follows from the orthogonality of the Haar system that

$$
\|b\|_{\mathrm{BMO}_{2}^{d}}=\left(\sup _{I} \frac{1}{|I|} \sum_{J \subseteq I} \frac{\left\langle b, h_{J}\right\rangle^{2}}{|J|}\right)^{1 / 2} .
$$

On $\mathbb{R}^{d}$, we may define $\mathrm{BMO}\left(\mathbb{R}^{d}\right)$ and its dyadic version in a similar way. 


\subsection{A key lemma}

The following lemma is quite useful and it provides a foundation for our analysis in the proof.

Lemma 2.2 (Fréchet-Kolmogorov-Riesz-Tsuji theorem, [15, 20]). Let $0<r<\infty$. A closed subset $\mathcal{K} \subseteq L^{r}$ is compact if and only if the following three conditions are satisfied:

(a) $\mathcal{K}$ is bounded in $L^{r}$;

(b) $\lim _{A \rightarrow \infty} \int_{|x|>A}|f(x)|^{r} d x=0$ uniformly for $f \in \mathcal{K}$;

(c) $\lim _{t \rightarrow 0}\|f(x+t)-f(x)\|_{L^{r}}=0$ uniformly for $f \in \mathcal{K}$.

\section{Proof of Theorem 1.1}

Now, we begin to prove Theorem 1.1 .

Proof. (i) Our first aim is to demonstrate the continuity of $\mathbb{S}(f)$. Let $|\nabla f|$ be bounded in $\mathbb{R}^{d}$. For $\varepsilon>0$, there exists $k_{0}>0$ such that $\sum_{k=k_{0}}^{\infty} \frac{1}{2^{k}}<\varepsilon$. Then, it holds that

$$
\lim _{t \rightarrow 0}|\mathbb{S} f(x+t)-\mathbb{S} f(x)| \leq I_{1}+I_{2}
$$

where

$$
\begin{aligned}
& I_{1}=\lim _{t \rightarrow 0}\left|\sum_{l(I) \leq 2^{-k_{0}}} \frac{1}{|I|} \int_{I} a_{I}(x+t, y) f(y) d y-\sum_{l(I) \leq 2^{-k_{0}}} \frac{1}{|I|} \int_{I} a_{I}(x, y) f(y) d y\right|, \\
& I_{2}=\lim _{t \rightarrow 0}\left|\sum_{l(I)>2^{-k_{0}}} \frac{1}{|I|} \int_{I} a_{I}(x+t, y) f(y) d y-\sum_{l(I)>2^{-k_{0}}} \frac{1}{|I|} \int_{I} a_{I}(x, y) f(y) d y\right| .
\end{aligned}
$$

Therefore, we need to consider the contributions of $I_{1}$ and $I_{2}$, respectively.

(1) Estimates for $I_{1}$. For any $x \in I$, there is only one cube $I^{\prime \prime}$ such that $x \in I^{\prime \prime}$. Hence, noting that $\left\|h_{I^{\prime}}\right\|_{\infty}\left\|h_{I^{\prime \prime}}\right\|_{\infty} \leq 1$, it yields that

$$
\begin{aligned}
\left|\frac{1}{|I|} \int_{I} a_{I}(x, y) f(y) d y\right| & =\left|\frac{1}{|I|} \sum_{\substack{I^{\prime}, I^{\prime \prime} \in \mathcal{D}, I^{\prime}, I^{\prime \prime} \subset I \\
l\left(I^{\prime}\right)=2^{-m} l(I), l\left(I^{\prime \prime}\right)=2^{-n} l(I)}}\left\langle f, h_{I^{\prime}}\right\rangle h_{I^{\prime \prime}}\right| \\
= & \left|\frac{1}{|I|} \sum_{\substack{I^{\prime} \subset I \\
l\left(I^{\prime}\right)=2^{-m} l(I)}} \sum_{J \in\left\{\operatorname{ch}\left(I^{\prime}\right)\right\}} \alpha_{J} \int_{J} f(y) d y h_{I^{\prime \prime}}\right| \\
\leq & \sum_{\substack{I^{\prime} \subset I \\
l\left(I^{\prime}\right)=2^{-m} l(I)}} \frac{1}{|I|}\left|\sum_{J \in\left\{\operatorname{ch}\left(I^{\prime}\right)\right\}} \frac{\alpha_{J}}{\left\|h_{I^{\prime}}\right\|_{\infty}} \int_{J} f(y) d y\right| .
\end{aligned}
$$


Let $x_{0} \in I^{\prime}$ be a fixed point. It is easy to see that $\sum_{J \in\left\{\operatorname{ch}\left(I^{\prime}\right)\right\}} \alpha_{J} f\left(x_{0}\right)|J|=0$. Then, the mean value theorem gives that

$$
\begin{aligned}
\left|\sum_{J \in\left\{\operatorname{ch}\left(I^{\prime}\right)\right\}} \frac{\alpha_{J}}{\left\|h_{I^{\prime}}\right\|_{\infty}} \int_{J} f(y) d y\right| & =\left|\sum_{J \in\left\{\operatorname{ch}\left(I^{\prime}\right)\right\}} \frac{\alpha_{J}}{\left\|h_{I^{\prime}}\right\|_{\infty}} \int_{J}\left(f(y)-f\left(x_{0}\right)\right) d y\right| \\
& \leq \sum_{J \in\left\{\operatorname{ch}\left(I^{\prime}\right)\right\}} \sqrt{d} l\left(I^{\prime}\right)\|\nabla f\|_{\infty}|J| \\
& \leq 2^{d \sqrt{d} 2^{-m(d+1)} l(I)|I| .}
\end{aligned}
$$

Consequently, this leads to

$$
\begin{aligned}
\left|\frac{1}{|I|} \int_{I} a_{I}(x, y) f(y) d y\right| & \leq \sum_{\substack{I^{\prime} \subset I \\
l\left(I^{\prime}\right)=2^{-m} l(I)}} \frac{1}{|I|} 2^{d} \sqrt{d} 2^{-m(d+1)} l(I)|I| \\
& \leq 2^{-m \sqrt{d}\|\nabla f\|_{\infty} l(I) .}
\end{aligned}
$$

Therefore, it holds that

$$
\begin{aligned}
& I_{1} \leq\left|\sum_{l(I) \leq 2^{-k_{0}}} \frac{1}{|I|} \int_{I} a_{I}(x+t, y) f(y) d y\right|+\left|\sum_{l(I) \leq 2^{-k_{0}}} \frac{1}{|I|} \int_{I} a_{I}(x, y) f(y) d y\right| \\
& \leq 2^{-m+1} \sqrt{d}\|\nabla f\|_{\infty} \sum_{l(I) \leq 2^{-k_{0}}} l(I) \\
& \lesssim \varepsilon .
\end{aligned}
$$

(2) Estimates for $I_{2}$. Let $\widetilde{\mathcal{D}}$ consist of all the boundary points of the dyadic cubes $I \in \mathcal{D}$. Let $x \in \mathbb{R}^{d} \backslash \widetilde{\mathcal{D}}$. Then there exists $I_{k_{0}} \in \mathcal{D}_{-k_{0}-m}$ such that $x \in I_{k_{0}}$. If $I \in \bigcup_{k=-k_{0}+1}^{\infty} \mathcal{D}_{k}$ contains $x$, then it follows that $x \in I_{k_{0}} \subset I$ and $I$ is an $\ell$-th ancestor of $I_{k_{0}}$ for $\ell \geq m$. Hence $I_{k_{0}}$ is contained in one of $\operatorname{ch}(I)$, which implies that $h_{I^{\prime \prime}}(x+t)=h_{I^{\prime \prime}}(x)$ for all $I \in \bigcup_{k=-k_{0}+1}^{\infty} \mathcal{D}_{k}$. Thus, it follows that

$$
\begin{aligned}
I_{2} & =\lim _{t \rightarrow 0}\left|\sum_{l(I)>2^{-k_{0}}} \frac{1}{|I|} \int_{I}\left(a_{I}(x+t, y)-a_{I}(x, y)\right) f(y) d y\right| \\
& \leq \lim _{t \rightarrow 0} \sum_{l(I)>2^{-k_{0}}} \frac{1}{|I|}\left|\sum_{\substack{I^{\prime}, I^{\prime \prime} \in \mathcal{D}, I^{\prime}, I^{\prime \prime} \subset I \\
l\left(I^{\prime}\right)=2^{-m} l(I), l\left(I^{\prime \prime}\right)=2^{-n} l(I)}}\left\langle f, h_{I^{\prime}}\right\rangle\left(h_{I^{\prime \prime}}(x+t)-h_{I^{\prime \prime}}(x)\right)\right| \\
& =\lim _{t \rightarrow 0} \sum_{l(I)>2^{-k_{0}}} \frac{1}{|I|}\left|\sum_{\substack{I^{\prime} \subset I \\
l\left(I^{\prime}\right)=2^{-m} l(I)}}\left\langle f, h_{I^{\prime}}\right\rangle\left(h_{I^{\prime \prime}}(x+t)-h_{I^{\prime \prime}}(x)\right)\right| \\
& =0 .
\end{aligned}
$$

Therefore, $\mathbb{S} f(x)$ is continuous almost everywhere. 
(ii) Now, we consider the continuity of $\pi_{b}^{\vec{\alpha}}(\vec{f})$. The proof of continuity for $T_{\epsilon}^{\vec{\alpha}}(\vec{f})$ follows similarly. Let $\vec{\alpha} \in\{0,1\}^{m} \backslash\{(1, \ldots, 1)\}$. Suppose that $f_{j}^{\prime}$ is bounded when $\alpha_{j}=0$ and $f_{j}$ is bounded when $\alpha_{j}=1$ in $\mathbb{R}$. For $\varepsilon>0$, there exists $k_{0}>0$ such that $\sum_{k=k_{0}}^{\infty} \frac{1}{2^{k}}<\varepsilon$. Then, it holds that

$$
\lim _{t \rightarrow 0}\left|\pi_{b}^{\vec{\alpha}}(\vec{f})(x+t)-\pi_{b}^{\vec{\alpha}}(\vec{f})(x)\right| \leq I I_{1}+I I_{2}
$$

where

$$
\begin{aligned}
I I_{1}=\lim _{t \rightarrow 0} \mid & \sum_{l(I) \leq 2^{-k_{0}}} \frac{\left\langle b, h_{I}\right\rangle}{|I|} \prod_{j=1}^{m} \frac{\left\langle f_{j}, h_{I}^{1+\alpha_{j}}\right\rangle}{|I|} h_{I}^{1+\sigma(\vec{\alpha})}(x+t) \\
& -\sum_{l(I) \leq 2^{-k_{0}}} \frac{\left\langle b, h_{I}\right\rangle}{|I|} \prod_{j=1}^{m} \frac{\left\langle f_{j}, h_{I}^{1+\alpha_{j}}\right\rangle}{|I|} h_{I}^{1+\sigma(\vec{\alpha})}(x) \mid
\end{aligned}
$$

and

$$
\begin{aligned}
I I_{2}=\lim _{t \rightarrow 0} \mid & \sum_{l(I)>2^{-k_{0}}} \frac{\left\langle b, h_{I}\right\rangle}{|I|} \prod_{j=1}^{m} \frac{\left\langle f_{j}, h_{I}^{1+\alpha_{j}}\right\rangle}{|I|} h_{I}^{1+\sigma(\vec{\alpha})}(x+t) \\
& -\sum_{l(I)>2^{-k_{0}}} \frac{\left\langle b, h_{I}\right\rangle}{|I|} \prod_{j=1}^{m} \frac{\left\langle f_{j}, h_{I}^{1+\alpha_{j}}\right\rangle}{|I|} h_{I}^{1+\sigma(\vec{\alpha})}(x) \mid .
\end{aligned}
$$

Next, we will estimate $I I_{1}$ and $I I_{2}$, respectively.

(1) Estimates for $I I_{1}$. For any $\alpha_{j}=0$, the mean value theorem yields that

$$
\frac{\left|\left\langle f_{j}, h_{I}\right\rangle\right|}{|I|}=\frac{\left|\int_{I_{+}}\left(f_{j}(x)-f_{j}\left(x_{I}\right)\right) d x-\int_{I_{-}}\left(f_{j}(x)-f\left(x_{I}\right)\right) d x\right|}{|I|} \leq|I| \sup _{x \in \mathbb{R}}\left|f_{j}^{\prime}(x)\right|,
$$

where $x_{I}$ is the center of the interval $I$. By the definition of $\mathrm{BMO}^{d}$, we know that $\frac{\left\langle b, h_{I}\right\rangle}{|I|}$ is bounded. The boundedness of $\frac{\left\langle f_{j}, h_{I}^{2}\right\rangle}{|I|}=\frac{\left\langle f_{j}, \chi_{I}\right\rangle}{|I|}$ follows from the boundedness of $f_{j}$ in $\mathbb{R}$. These basic facts yield that

$$
\begin{aligned}
I I_{1} \leq & \left|\sum_{l(I) \leq 2^{-k_{0}}} \frac{\left\langle b, h_{I}\right\rangle}{|I|} \prod_{j=1}^{m} \frac{\left\langle f_{j}, h_{I}^{1+\alpha_{j}}\right\rangle}{|I|} h_{I}^{1+\sigma(\vec{\alpha})}(x+t)\right| \\
& +\left|\sum_{l(I) \leq 2^{-k_{0}}} \frac{\left\langle b, h_{I}\right\rangle}{|I|} \prod_{j=1}^{m} \frac{\left\langle f_{j}, h_{I}^{1+\alpha_{j}}\right\rangle}{|I|} h_{I}^{1+\sigma(\vec{\alpha})}(x)\right| \\
& \leq 2 \sum_{l(I) \leq 2^{-k_{0}}}\left|\frac{\left\langle b, h_{I}\right\rangle}{|I|} \prod_{\alpha_{j}=0} \frac{\left\langle f_{j}, h_{I}\right\rangle}{|I|} \prod_{\alpha_{j}=1} \frac{\left\langle f_{j}, \chi_{I}\right\rangle}{|I|}\right| \\
& \lesssim 2\|b\|_{\mathrm{BMO}} \sum_{k=k_{0}}^{\infty}\left(\frac{1}{2^{k}}\right)^{\sigma(\vec{\alpha})} \\
& \lesssim \varepsilon
\end{aligned}
$$


(2) Estimates for $I I_{2}$. Let $\widetilde{\mathcal{D}}$ consist of all end-points of the dyadic intervals $I \in \mathcal{D}$. Let $x \in \widetilde{\mathcal{D}}^{c}$. Then there exists $I_{k_{0}} \in \mathcal{D}_{-k_{0}}$ such that $x \in I_{k_{0}}$. If $I \in \bigcup_{k=-k_{0}+1}^{\infty} \mathcal{D}_{k}$ contains $x$, then $I_{k_{0}}$ is contained in either $I_{+}$or $I_{-}$, which implies $h_{I}(x+t)=h_{I}(x)$ for $|t|<\operatorname{dist}\left(x, I_{k_{0}}^{c}\right)$. Therefore, for $I \in \bigcup_{k=-k_{0}+1}^{\infty} \mathcal{D}_{k}$ and $x \in I$ we get $h_{I}(x+t)=h_{I}(x)$. Then $h_{I}^{1+\sigma(\vec{\alpha})}(x+t)-h_{I}^{1+\sigma(\vec{\alpha})}(x)=0$. Consequently, it holds that

$$
I I_{2}=\lim _{t \rightarrow 0}\left|\sum_{l(I)>2^{-k_{0}}} \frac{\left\langle b, h_{I}\right\rangle}{|I|} \prod_{j=1}^{m} \frac{\left\langle f_{j}, h_{I}^{1+\alpha_{j}}\right\rangle}{|I|}\left(h_{I}^{1+\sigma(\vec{\alpha})}(x+t)-h_{I}^{1+\sigma(\vec{\alpha})}(x)\right)\right|=0 .
$$

Finally, for $\vec{\alpha} \in\{0,1\}^{m} \backslash\{(1, \ldots, 1)\}$, we have showed that $\pi_{b}^{\vec{\alpha}}(\vec{f})(x)$ is continuous almost everywhere. When $\vec{\alpha}=(1,1, \ldots, 1)$, let $b^{\prime}(x)$ be bounded, proceeding with similar arguments as before, one may obtain that $\pi_{b}^{\vec{\alpha}}(\vec{f})(x)$ is almost everywhere continuous for all bounded $f_{j}$ in $\mathbb{R}$.

\section{Proofs of Theorems 1.3 and 1.4}

Proof of Theorem 1.3. Let $T$ be any of these dyadic operators and $\mathcal{K}=\{T(\vec{f})(x)$ : $\left.\left\|f_{j}\right\|_{L^{p_{j}}} \leq 1, j=1, \ldots, m\right\}$. According to the definition of compact operator, we need to show that $\mathcal{K}$ is precompact ( $\overline{\mathcal{K}}$ is compact). It is obvious that $T_{\left\{\epsilon_{I}=1\right\}}^{\{0\}}$ is the identity operator on $L^{p}(\mathbb{R})$ by the reason that $\sum_{I \in \mathcal{D}}\left\langle f, h_{I}\right\rangle h_{I}=f(x)$ for $f \in L^{p}(\mathbb{R})(1<p<\infty)$. Moreover, $T_{\left\{\epsilon_{I}=1\right\}}^{\{0\}}$ is not a compact operator since the unit ball of $L^{p}(\mathbb{R})$ is not a compact set. Counter-examples will be given to illustrate that $\mathcal{K}$ doesn't satisfy the condition (c) for any Haar multipliers and dyadic shift, which implies the noncompactness of these dyadic operators. (i) By the Fréchet-Kolmogorov-Riesz-Tsuji theorem, we need to show that

$$
\mathcal{K}=\left\{T_{\epsilon}^{\vec{\alpha}}(\vec{f})(x):\left\|f_{j}\right\|_{p_{j}} \leq 1\right\}
$$

does't meet at least one of the three conditions.

We first observe the following: For $I \in \mathcal{D}$, we define $\vec{f}_{I}=\left(f_{I, 1}, \ldots, f_{I . m}\right)$ by $f_{I, j}=$ $|I|^{-1 / p_{j}} h_{I}^{1+\alpha_{j}}$. Then we have

$$
\left\|f_{I, j}\right\|_{p_{j}}=1, \quad\left\langle f_{I, j}, h_{I}^{1+\alpha_{j}}\right\rangle=|I|^{1-1 / p_{j}}, \quad\left\langle f_{I, j}, h_{J}\right\rangle=0 \quad \text { for } I \neq J \in \mathcal{D}, \alpha_{j}=0 .
$$

Hence, noting $\alpha_{j}=0$ for at least one $1 \leq j \leq m$, we get

$$
T_{\epsilon}^{\vec{\alpha}}\left(\vec{f}_{I}\right)=\epsilon_{I}|I|^{-1 / p} h_{I}^{\sigma(\vec{\alpha})},
$$

and so

$$
\left\|T_{\epsilon}^{\vec{\alpha}}\left(\vec{f}_{I}\right)\right\|_{p}=\left|\epsilon_{I}\right|
$$


For $|I|<t$, we have $(I+t) \cap I=\emptyset$, and hence

$$
\left\|T_{\epsilon}^{\vec{\alpha}}\left(\overrightarrow{f_{I}}\right)(x+t)-T_{\epsilon}^{\vec{\alpha}}\left(\overrightarrow{f_{I}}\right)(x)\right\|_{p}=2\left\|T_{\epsilon}^{\vec{\alpha}}\left(\vec{f}_{I}\right)\right\|_{p}=2\left|\epsilon_{I}\right|
$$

Next, suppose that there exists $A>0$ such that $\#\left\{I \in \mathcal{D}:\left|\epsilon_{I}\right| \geq A\right\}=\infty$. We consider the following two cases: (1) $A_{1}:=\lim _{k \rightarrow \infty} \sup _{I \in \mathcal{D}, I \subset\left[2^{k}, \infty\right) \cup\left(-\infty,-2^{k}\right)}\left|\epsilon_{I}\right|>0$, and (2) $\lim _{k \rightarrow \infty} \sup _{I \in \mathcal{D}, I \subset\left[2^{k}, \infty\right) \cup\left(-\infty,-2^{k}\right)}\left|\epsilon_{I}\right|=0$.

(1) In this case, by 4.1 and 4.2 we see that

$$
\limsup _{B \rightarrow \infty} \sup _{\left\|f_{I, j}\right\|_{p_{j}} \leq 1,1 \leq j \leq m}\left(\int_{|x| \geq B}\left|T_{\epsilon}^{\vec{\alpha}}\left(\vec{f}_{I}\right)(x)\right|^{p} d x\right)^{1 / p} \geq A_{1}>0,
$$

which shows the condition (b) does not hold.

(2) In this case, there exists $k_{0} \in \mathbb{N}$ such that $\#\left\{I \in \mathcal{D}, I \in\left[-2^{k_{0}}, 2^{k_{0}}\right]:\left|\epsilon_{I}\right| \geq A\right\}=\infty$, from which it follows that there exists $I_{k} \in \mathcal{D}$ such that $\left|\epsilon_{I_{k}}\right| \geq A, I_{k} \subset\left[-2^{k_{0}}, 2^{k_{0}}\right]$ and $\lim _{k \rightarrow \infty}\left|I_{k}\right|=0$. Hence, by (4.1) and (4.3), it follows that

$$
\limsup _{t \rightarrow 0} \sup _{\left\|f_{I, j}\right\|_{p_{j}} \leq 1,1 \leq j \leq m}\left\|T_{\epsilon}^{\vec{\alpha}}\left(\vec{f}_{I}\right)(x+t)-T_{\epsilon}^{\vec{\alpha}}\left(\vec{f}_{I}\right)(x)\right\|_{p} \geq 2 A>0
$$

This shows that condition (c) does not hold.

Hence, in any case, by the Fréchet-Kolmogorov-Riesz-Tsuji theorem, we know that $T_{\epsilon}^{\vec{\alpha}}(\vec{f})$ is not compact, under our assumption.

(ii) Suppose that $\mathbb{S}$ is a dyadic shift with parameter $(m, n)$. Then, we can show that dyadic shift operator is not compact in the same way as in the case of $T_{\epsilon}^{\vec{\alpha}}$. We omit the proof of it.

Proof of Theorem 1.4. Firstly, we recall the boundedness result of $\pi_{b}^{\vec{\alpha}}$. Since

$$
\sup _{I \in \mathcal{D}} \frac{\left|\left\langle b, h_{I}\right\rangle\right|}{|I|} \leq\left(\sup _{I \in \mathcal{D}} \frac{1}{|I|} \sum_{J \in \mathcal{D}, J \subset I} \frac{\left|\left\langle b, h_{I}\right\rangle\right|^{2}}{|I|}\right)^{1 / 2}=\|b\|_{\mathrm{BMO}_{2}^{d}} \leq\|b\|_{\mathrm{BMO}}
$$

(as pointed out by Kunwar [8, Page 14]) by Theorem 3.7 in $9 \pi_{b}^{\vec{\alpha}}$ is a bounded operator from $L^{p_{1}} \times \cdots \times L^{p_{m}}$ to $L^{p}$, provided $b \in \mathrm{BMO}, 1<p_{1}, \ldots, p_{m}<\infty$ and $1 / p_{1}+\cdots+1 / p_{m}=$ $1 / p$.

We now proceed to show the compactness of $\pi_{b}^{\vec{\alpha}}$.

(i) The case $b \in C_{c}^{\infty}(\mathbb{R})$. By the boundedness of $\pi_{b}^{\vec{\alpha}}$, it is trivial that $\pi_{b}^{\vec{\alpha}}$ satisfies the condition (a). Now we verify the condition (b) and the condition (c) for its compactness. We may assume $b \in C_{c}^{\infty}(\mathbb{R})$ with $\operatorname{supp} b \subset(-1,1)$. For $k \geq 1$, the supports of $b$ and $h_{I}$ 
give that

$$
\begin{aligned}
& \int_{|x| \geq 2^{k}}\left|\pi_{b}^{\vec{\alpha}}(\vec{f})(x)\right|^{p} d x \\
= & \int_{|x| \geq 2^{k}}\left|\sum_{I \in \mathcal{D}} \frac{\left\langle b, h_{I}\right\rangle}{|I|} \prod_{j=1}^{m} \frac{\left\langle f_{j}, h_{I}^{1+\alpha_{j}}\right\rangle}{|I|} h_{I}^{1+\sigma(\vec{\alpha})}(x)\right|^{p} d x \\
= & \left.\int_{|x| \geq 2^{k}} \sum_{I=\left[0,2^{\ell}\right),\left[-2^{\ell}, 0\right), \ell \geq k} \frac{\left\langle b, h_{I}\right\rangle}{|I|} \prod_{j=1}^{m} \frac{\left\langle f_{j}, h_{I}^{1+\alpha_{j}}\right\rangle}{|I|} h_{I}^{1+\sigma(\vec{\alpha})}(x)\right|^{p} d x \\
\leq & \int_{|x| \geq 2^{k}}\left(\sum_{I=\left[0,2^{\ell}\right),\left[-2^{\ell}, 0\right), \ell \geq k}|| b\left\|_{\infty} \frac{2}{|I|} \prod_{j=1}^{m}\right\| f_{j} \|_{p_{j}} \frac{|I|^{1 / p_{j}^{\prime}}}{|I|} \chi_{I}(x)\right)^{p} d x \\
\leq & C\left(\|b\|_{\infty} \prod_{1}^{m}\left\|f_{j}\right\|_{p_{j}}\right)^{p} \int_{|x| \geq 2^{k}}\left(\sum_{I=\left[0,2^{\ell}\right),\left[-2^{\ell}, 0\right), \ell \geq k}|I|^{-1 / p-1} \chi_{I}(x)\right)^{p} d x \\
\leq & C\left(\|b\|_{\infty} \prod_{1}^{m}\left\|f_{j}\right\|_{p_{j}}\right)^{p} \int_{|x| \geq 2^{k}}\left(\sum_{\ell \geq k} 2^{-\ell(1 / p+1)} \chi_{\left[-2^{\ell}, 2^{\ell}\right)}(x)\right)^{p} d x \\
\leq & C\left(\|b\|_{\infty} \prod_{1}^{m}\left\|f_{j}\right\|_{p_{j}}\right)^{p} \sum_{\ell=k}^{\infty} \int_{2^{\ell}}^{2^{\ell+1}}\left(2^{-\ell(1 / p+1)}\right)^{p} d x \\
= & C\left(\|b\|_{\infty} \prod_{1}^{m}\left\|f_{j}\right\|_{p_{j}}\right)^{p} \sum_{\ell=k}^{\infty} 2^{-p \ell}=C\left(\|b\|_{\infty} \prod_{1}^{m}\left\|f_{j}\right\|_{p_{j}}\right)^{p} 2^{-p k} .
\end{aligned}
$$

Hence we have

$$
\lim _{A \rightarrow \infty} \int_{|x| \geq A}\left|\pi_{b}^{\vec{\alpha}}(\vec{f})(x)\right|^{p} d x=0
$$

uniformly for $\vec{f}$ with $\left\|f_{j}\right\|_{p_{j}} \leq 1(1 \leq j \leq m)$. Consequently, when $b \in C_{c}^{\infty}(\mathbb{R}), \pi_{b}^{\vec{\alpha}}$ satisfies the condition (b) for its compactness.

Let $1<p_{1}, \ldots, p_{m}<\infty$ and $1 / p=1 / p_{1}+\cdots+1 / p_{m}$. Now, we only need to consider dyadic intervals $I$ with $(-1,1) \cap I \neq \emptyset$ in the following summation. Therefore, it holds that

$$
\begin{aligned}
& \left(\left.\int \frac{\left\langle b, h_{I}\right\rangle}{|I|} \prod_{j=1}^{m} \frac{\left\langle f_{j}, h_{I}^{1+\alpha_{j}}\right\rangle}{|I|}\left(h_{I}^{1+\sigma(\vec{\alpha})}(x+h)-h_{I}^{1+\sigma(\vec{\alpha})}(x)\right)\right|^{p} d x\right)^{1 / p} \\
\leq & \left(\int\|b\|_{\infty} \prod_{j=1}^{m} \frac{\left\|f_{j}\right\|_{p_{j}}|I|^{1 / p_{j}^{\prime}}}{|I|}\left|\left(h_{I}^{1+\sigma(\vec{\alpha})}(x+h)-h_{I}^{1+\sigma(\vec{\alpha})}(x)\right)\right|^{p} d x\right)^{1 / p} \\
\leq & C\|b\|_{\infty} \prod_{j=1}^{m}\left\|f_{j}\right\|_{p_{j}} \frac{|h|^{1 / p}}{|I|^{1 / p}} .
\end{aligned}
$$


Thus we get

$$
\begin{aligned}
& \left(\int\left|\sum_{|I| \geq 1} \frac{\left\langle b, h_{I}\right\rangle}{|I|} \prod_{j=1}^{m} \frac{\left\langle f_{j}, h_{I}^{1+\alpha_{j}}\right\rangle}{|I|}\left(h_{I}^{1+\sigma(\vec{\alpha})}(x+h)-h_{I}^{1+\sigma(\vec{\alpha})}(x)\right)\right|^{p} d x\right)^{1 / p} \\
\leq & \sum_{|I| \geq 1}\left(\int\left|\frac{\left\langle b, h_{I}\right\rangle}{|I|} \prod_{j=1}^{m} \frac{\left\langle f_{j}, h_{I}^{1+\alpha_{j}}\right\rangle}{|I|}\left(h_{I}^{1+\sigma(\vec{\alpha})}(x+h)-h_{I}^{1+\sigma(\vec{\alpha})}(x)\right)\right|^{p} d x\right)^{1 / p} \\
\leq & C\|b\|_{\infty} \prod_{j=1}^{m}\left\|f_{j}\right\|_{p_{j}} \sum_{I=\left[0,2^{\ell}\right),\left[-2^{\ell}, 0\right), \ell \in \mathbb{N}} \frac{|h|^{1 / p}}{|I|^{1 / p}} \leq C\|b\|_{\infty} \prod_{j=1}^{m}\left\|f_{j}\right\|_{p_{j}}|h|^{1 / p} .
\end{aligned}
$$

Next, for $|h| \leq|I|$, noting that $1 / p=1 / p_{1}+\cdots+1 / p_{m}$ and $\int h_{I} d x=0$, we have

$$
\begin{aligned}
& \left(\int\left|\frac{\left\langle b, h_{I}\right\rangle}{|I|} \prod_{j=1}^{m} \frac{\left\langle f_{j}, h_{I}^{1+\alpha_{j}}\right\rangle}{|I|}\left(h_{I}^{1+\sigma(\vec{\alpha})}(x+h)-h_{I}^{1+\sigma(\vec{\alpha})}(x)\right)\right|^{p} d x\right)^{1 / p} \\
= & \left(\int\left|\frac{\left\langle b-b\left(x_{I}\right), h_{I}\right\rangle}{|I|} \prod_{j=1}^{m} \frac{\left\langle f_{j}, h_{I}^{1+\alpha_{j}}\right\rangle}{|I|}\left(h_{I}^{1+\sigma(\vec{\alpha})}(x+h)-h_{I}^{1+\sigma(\vec{\alpha})}(x)\right)\right|^{p} d x\right)^{1 / p} \\
\leq & \left\|b^{\prime}\right\|_{\infty}|I| \prod_{j=1}^{m} \frac{\left|\left\langle f_{j}, h_{I}^{1+\alpha_{j}}\right\rangle\right|}{|I|}\left(\int\left|h_{I}^{1+\sigma(\vec{\alpha})}(x+h)-h_{I}^{1+\sigma(\vec{\alpha})}(x)\right|^{p} d x\right)^{1 / p} \\
\leq & C\left\|b^{\prime}\right\|_{\infty} \prod_{j=1}^{m}\left\|f_{j} \chi_{I}\right\|_{p_{j}}|h|^{1 / p}|I|^{1-1 / p},
\end{aligned}
$$

where $x_{I}$ is the center of the dyadic interval $I$.

Similarly, in the case $|I| \leq|h|$, it holds that $\left(\int\left|\left(h_{I}^{1+\sigma(\vec{\alpha})}(x+h)-h_{I}^{1+\sigma(\vec{\alpha})}(x)\right)\right|^{p} d x\right)^{1 / p} \leq$ $C|I|^{1 / p}$. Then, we may also obtain

$$
\left(\int\left|\frac{\left\langle b, h_{I}\right\rangle}{|I|} \prod_{j=1}^{m} \frac{\left\langle f_{j}, h_{I}^{1+\alpha_{j}}\right\rangle}{|I|}\left(h_{I}^{1+\sigma(\vec{\alpha})}(x+h)-h_{I}^{1+\sigma(\vec{\alpha})}(x)\right)\right|^{p} d x\right)^{1 / p} \leq C\left\|b^{\prime}\right\|_{\infty} \prod_{j=1}^{m}\left\|f_{j} \chi_{I}\right\|_{p_{j}}|I| .
$$

So, for any $0<a<1$, we have

$$
\begin{aligned}
& \left(\int\left|\frac{\left\langle b, h_{I}\right\rangle}{|I|} \prod_{j=1}^{m} \frac{\left\langle f_{j}, h_{I}^{1+\alpha_{j}}\right\rangle}{|I|}\left(h_{I}^{1+\sigma(\vec{\alpha})}(x+h)-h_{I}^{1+\sigma(\vec{\alpha})}(x)\right)\right|^{p} d x\right)^{1 / p} \\
\leq & C\left\|b^{\prime}\right\|_{\infty} \prod_{j=1}^{m}\left\|f_{j} \chi_{I}\right\|_{p_{j}}|h|^{a}|I|^{1-a} .
\end{aligned}
$$

Thus, when $p>1$, for every $\ell \in \mathbb{N}$, we get

$$
\begin{aligned}
& \sum_{|I|=2^{-\ell}}\left(\int\left|\frac{\left\langle b, h_{I}\right\rangle}{|I|} \prod_{j=1}^{m} \frac{\left\langle f_{j}, h_{I}^{1+\alpha_{j}}\right\rangle}{|I|}\left(h_{I}^{1+\sigma(\vec{\alpha})}(x+h)-h_{I}^{1+\sigma(\vec{\alpha})}(x)\right)\right|^{p} d x\right)^{1 / p} \\
\leq & C\left\|b^{\prime}\right\|_{\infty}|h|^{1 / p} \sum_{|I|=2^{-\ell}}|I|^{1-1 / p} \prod_{j=1}^{m}\left\|f_{j} \chi_{I}\right\|_{p_{j}}
\end{aligned}
$$




$$
\begin{aligned}
& \leq C\left\|b^{\prime}\right\|_{\infty}|h|^{1 / p} 2^{-(1-1 / p) \ell} \prod_{j=1}^{m}\left(\sum_{|I|=2^{-\ell}}\left\|f_{j} \chi_{I}\right\|_{p_{j}}^{p_{j}}\right)^{1 / p_{j}} \\
& \leq C\left\|b^{\prime}\right\|_{\infty}|h|^{1 / p} 2^{-(1-1 / p) \ell} \prod_{j=1}^{m}\left\|f_{j}\right\|_{p_{j}} .
\end{aligned}
$$

This leads to the following estimate:

$$
\begin{aligned}
& \left(\int\left|\sum_{|I|<1, I \cap(-1,1) \neq \emptyset} \frac{\left\langle b, h_{I}\right\rangle}{|I|} \prod_{j=1}^{m} \frac{\left\langle f_{j}, h_{I}^{1+\alpha_{j}}\right\rangle}{|I|}\left(h_{I}^{1+\sigma(\vec{\alpha})}(x+h)-h_{I}^{1+\sigma(\vec{\alpha})}(x)\right)\right|^{p} d x\right)^{1 / p} \\
\leq & C\left\|b^{\prime}\right\|_{\infty} \prod_{j=1}^{m}\left\|f_{j}\right\|_{p_{j}}|h|^{1 / p} .
\end{aligned}
$$

When $p \leq 1$ and $|h|<|I|$, it is easy to see that $|h|^{1 / p}|I|^{1-1 / p}<|h|^{a}|I|^{1-a}$ for some $0<a<1$. Therefore, when $|h|<|I|<1$, for some $0<a<1$, we have

$$
\begin{aligned}
& \left(\int\left|\frac{\left\langle b, h_{I}\right\rangle}{|I|} \prod_{j=1}^{m} \frac{\left\langle f_{j}, h_{I}^{1+\alpha_{j}}\right\rangle}{|I|}\left(h_{I}^{1+\sigma(\vec{\alpha})}(x+h)-h_{I}^{1+\sigma(\vec{\alpha})}(x)\right)\right|^{p} d x\right)^{1 / p} \\
\leq & C\left\|b^{\prime}\right\|_{\infty} \prod_{j=1}^{m}\left\|f_{j} \chi_{I}\right\|_{p_{j}}|h|^{a}|I|^{1-a} .
\end{aligned}
$$

Consequently, when $p \leq 1$, by modifying a little bit, for some $0<a<1$, we get

$$
\begin{aligned}
& \left(\int\left|\sum_{|I|<1, I \cap(-1,1) \neq \emptyset} \frac{\left\langle b, h_{I}\right\rangle}{|I|} \prod_{j=1}^{m} \frac{\left\langle f_{j}, h_{I}^{1+\alpha_{j}}\right\rangle}{|I|}\left(h_{I}^{1+\sigma(\vec{\alpha})}(x+h)-h_{I}^{1+\sigma(\vec{\alpha})}(x)\right)\right|^{p} d x\right)^{1 / p} \\
\leq & C\left\|b^{\prime}\right\|_{\infty} \prod_{j=1}^{m}\left\|f_{j}\right\|_{p_{j}}|h|^{a} .
\end{aligned}
$$

Thus, we obtain

$$
\lim _{h \rightarrow 0}\left\|\pi_{b}^{\vec{\alpha}}(x+h)-\pi_{b}^{\vec{\alpha}}(x)\right\|_{p}=0
$$

uniformly for $\vec{f}$ with $\left\|f_{j}\right\|_{p_{j}} \leq 1(j=1, \ldots, m)$. This shows that $\pi_{b}^{\vec{\alpha}}$ satisfies the condition (c).

Hence, by the Fréchet-Kolmogorov-Riesz-Tsuji theorem, it follows that $\pi_{b}^{\vec{\alpha}}$ is a compact operator, provided $b \in C_{c}^{\infty}(\mathbb{R})$.

(ii) The general case $b \in \mathrm{CMO}(\mathbb{R})$. Since $C_{c}^{\infty}(\mathbb{R})$ is dense in $\mathrm{CMO}(\mathbb{R})$ in BMO norm, for any $b \in \mathrm{CMO}$ we can approximate it by a sequence of $C_{c}^{\infty}(\mathbb{R})$ functions $b_{j}$ in BMO norm. So, by the case (i) $\pi_{b}^{\vec{\alpha}}$ can be approximated arbitrarily near in operator norm by compact operators $\pi_{b_{j}}^{\vec{\alpha}}$, and hence it follows that $\pi_{b}^{\vec{\alpha}}$ itself is a compact operator. This procedure is used by many authors, but we can find no references we shall give a corresponding lemma and its proof in the appendix, for the sake of completeness. 
Remark 4.1. The condition that $b \in \mathrm{CMO}$ is necessary by the reason that there exists $b \in L^{\infty} \subset \mathrm{BMO}$ such that $\pi_{b}^{\vec{\alpha}}$ is not a compact operator. To show this, we will construct an example. Let $k_{0} \in \mathbb{N}$ and $t \in\left[2^{-k_{0}}, 3 \cdot 2^{-k_{0}+1}\right)$. Suppose that

$$
b=\sum_{k=1}^{\infty}(-1)^{k} \chi_{\left[1-2 / 2^{k}+1 / 2^{k+1}, 1-1 / 2^{k}\right)}
$$

and

$$
f(x)=f_{k_{0}}(x)= \begin{cases}-2^{k_{0}} & \text { when } x \in \frac{1}{2^{k_{0}}}\left([0,1)+2^{k_{0}}-2\right), \\ 0 & \text { otherwise. }\end{cases}
$$

We assume that $0^{0}=0$ and $f_{j}=f^{1-\alpha_{j}}$. Then one can verify that

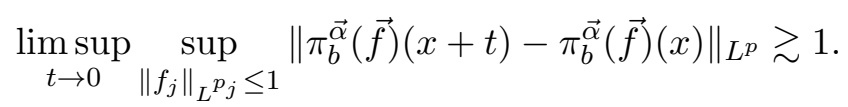

\section{Proof of Theorem 1.5}

To begin with, we need to consider the strong type boundedness of these commutators. From [8], we know that the commutators in the $j$-th entry are bounded from $L^{p_{1}} \times L^{p_{2}} \times$ $\cdots \times L^{p_{m}} \rightarrow L^{p}$, if $b \in$ BMO. Naturally, we ought to study the boundedness of iterated commutators and we obtain the following lemmas.

Lemma 5.1 (Weighted strong bounds for $\left.T_{\epsilon, \Pi \mathbf{b}}^{\vec{\alpha}}\right)$. Let $\vec{p}=\left(p_{1}, \ldots, p_{m}\right)$ with $\frac{1}{p}=\frac{1}{p_{1}}+\cdots+$ $\frac{1}{p_{m}}$ and $1<p_{1}, \ldots, p_{m}<\infty$. Let $\vec{\alpha} \in\{0,1\}^{m} \backslash\{1, \ldots, 1\}$ and $\epsilon=\left\{\epsilon_{I}\right\}_{I \in \mathcal{D}}$ be bounded. Suppose that $\vec{b}=\left(b_{1}, \ldots, b_{m}\right) \in\left(\mathrm{BMO}^{d}\right)^{m}, \vec{\omega} \in A_{\vec{p}}^{d}$ and $\nu_{\vec{\omega}}=\prod_{j=1}^{m} \omega_{j}^{p / p_{j}}$. Then there exists a constant $C$ such that

$$
\left\|T_{\epsilon, \Pi \mathbf{b}}^{\vec{\alpha}}\right\|_{L^{p}\left(\nu_{\vec{\omega}}\right)} \leq C \prod_{j=1}^{m}\left\|b_{j}\right\|_{\mathrm{BMO}_{d}} \prod_{i=1}^{m}\left\|f_{i}\right\|_{L^{p_{i}\left(\omega_{i}\right)}} .
$$

Lemma 5.2 (Weighted end-point estimate for $T_{\epsilon, \Pi \mathbf{b}}^{\vec{\alpha}}$ ). Let $\vec{\alpha} \in\{0,1\}^{m} \backslash\{1, \ldots, 1\}$ and $\epsilon=\left\{\epsilon_{I}\right\}_{I \in \mathcal{D}}$ be bounded. Suppose $\vec{b}=\left(b_{1}, \ldots, b_{m}\right) \in \mathrm{BMO}_{d}^{m}, \vec{\omega} \in A_{(1, \ldots, 1)}^{d}$ and $\nu_{\vec{\omega}}=$ $\prod_{j=1}^{m} \omega_{j}^{p / p_{j}}$. Then there exists a constant $C$ such that

$$
\nu_{\vec{\omega}}\left(x \in \mathbb{R}: T_{\epsilon, \Pi \mathbf{b}}^{\vec{\alpha}}(\vec{f})(x)>t^{m}\right) \leq C\left(\prod_{j=1}^{m} \Phi\left(\frac{\left|f_{j}(x)\right|}{t}\right) \omega_{j}(x) d x\right)^{\frac{1}{m}}
$$

where $\Phi(t)=t\left(1+\log ^{+} t\right)$ and $\Phi^{(m)}=\overbrace{\Phi \circ \cdots \circ \Phi}^{m}$.

The ideas and main steps of proofs for Lemmas 5.1 and 5.2 are almost the same as in 13, 19]. Moreover, Lemma 3.1 of [8] makes the proofs much easier. Here we omit the proofs.

Now we return to the proof of Theorem 1.5 . 
Proof of Theorem 1.5. (i) First, we shall prove the compactness of the commutator $\left[b, T_{\epsilon}^{\vec{\alpha}}\right]_{i}$. By its boundedness, verification of condition (a) is trivial and we will only prove that $\left[b, T_{\epsilon}^{\vec{\alpha}}\right]_{i}$ satisfies conditions (b) and (c) for its compactness. Firstly, we may assume $b \in C_{c}^{\infty}(\mathbb{R})$ with $\operatorname{supp} b \subset(-1,1)$ and $f_{j} \in L^{p_{j}}(\mathbb{R})\left(1<p_{j}<\infty\right)$. For $k \geq 1$, by the supports of $b$ and $h_{I}$, it holds that

$$
\begin{aligned}
& \int_{|x| \geq 2^{k}}\left|\left[b, T_{\epsilon}^{\vec{\alpha}}\right]_{i}(\vec{f})(x)\right|^{p} d x \\
= & \int_{|x| \geq 2^{k}}\left|\sum_{I=\left[0,2^{\ell}\right),\left[-2^{\ell}, 0\right), \ell \geq 1} \epsilon_{I} \frac{\left\langle b f_{i}, h_{I}^{1+\alpha_{i}}\right\rangle}{|I|} \prod_{1 \leq j \leq m, j \neq i} \frac{\left\langle f_{j}, h_{I}^{1+\alpha_{j}}\right\rangle}{|I|} h_{I}^{\sigma(\vec{\alpha})}(x)\right|^{p} d x \\
\leq & C \int_{|x| \geq 2^{k}}\left(\sum_{I=\left[0,2^{\ell}\right),\left[-2^{\ell}, 0\right), \ell \geq k}\left|\epsilon_{I}\right|\|b\|_{\infty} \frac{\left\|f_{i} \chi_{(-1,1)}\right\|_{p_{i}}}{|I|} \prod_{1 \leq j \leq m, j \neq i}\left\|f_{j}\right\|_{p_{j}} \frac{|I|^{1 / p_{j}^{\prime}}}{|I|} \chi_{I}(x)\right)^{p} d x \\
\leq & C\left(\|\epsilon\|_{\infty}\|b\|_{\infty} \prod_{j=1}^{m}\left\|f_{j}\right\|_{p_{j}}\right)^{p} \int_{|x| \geq 2^{k}}\left(\sum_{I=\left[0,2^{\ell}\right),\left[-2^{\ell}, 0\right), \ell \geq k}|I|^{-1 / p-1+1 / p_{i}} \chi_{I}(x)\right)^{p} d x \\
\leq & C\left(\|\epsilon\|_{\infty}\|b\|_{\infty} \prod_{j=1}^{m}\left\|f_{j}\right\|_{p_{j}}\right)^{p} \int_{|x| \geq 2^{k}}\left(\sum_{\ell \geq k} 2^{-\ell\left(1 / p+1 / p_{i}^{\prime}\right)} \chi_{\left[-2^{\ell}, 2^{\ell}\right)}(x)\right)^{p} d x \\
\leq & C\left(\|\epsilon\|_{\infty}\|b\|_{\infty} \prod_{j=1}^{m}\left\|f_{j}\right\|_{p_{j}}\right)^{p} \sum_{\ell=k}^{\infty} \int_{2^{\ell}}^{2^{\ell+1}}\left(2^{-\ell\left(1 / p+1 / p_{i}^{\prime}\right)}\right)^{p} d x \\
= & C\left(\|\epsilon\|_{\infty}\|b\|_{\infty} \prod_{j=1}^{m}\left\|f_{j}\right\|_{p_{j}}\right)^{p} \sum_{\ell=k}^{\infty} 2^{-\ell p / p_{i}^{\prime}} \\
= & C\left(\|\epsilon\|_{\infty}\|b\|_{\infty} \prod_{j=1}^{m}\left\|f_{j}\right\|_{p_{j}}\right)^{p} 2^{-k p / p_{i}^{\prime}} .
\end{aligned}
$$

Hence we have

$$
\lim _{A \rightarrow \infty} \int_{|x| \geq A}\left|\left[b, T_{\epsilon}^{\vec{\alpha}}\right]_{i}(\vec{f})(x)\right|^{p} d x=0
$$

uniformly for $\vec{f}$ with $\left\|f_{j}\right\|_{p_{j}} \leq 1(1 \leq j \leq m)$. Consequently, when $b \in \mathrm{CMO},\left[b, T_{\epsilon}^{\vec{\alpha}}\right]_{i}$ satisfies the condition (b) for any $1 \leq i \leq m$ and $\vec{\alpha} \in\{0,1\}^{m} \backslash\{1, \ldots, 1\}$.

Let $|h|<1$. We can rewrite $\left[b, T_{\epsilon}^{\vec{\alpha}}\right]_{i}(\vec{f})(x+h)-\left[b, T_{\epsilon}^{\vec{\alpha}}\right]_{i}(\vec{f})(x)$ in the following way

$$
\begin{aligned}
& {\left[b, T_{\epsilon}^{\vec{\alpha}}\right]_{i}(\vec{f})(x+h)-\left[b, T_{\epsilon}^{\vec{\alpha}}\right]_{i}(\vec{f})(x) } \\
= & b(x+h) T_{\epsilon}^{\vec{\alpha}}(\vec{f})(x+h)-T_{\epsilon}^{\vec{\alpha}}\left(f_{1}, \ldots, b f_{i}, f_{i+1}, \ldots, f_{m}\right)(x+h) \\
& -b(x) T_{\epsilon}^{\vec{\alpha}}(\vec{f})(x)+T_{\epsilon}^{\vec{\alpha}}\left(f_{1}, \ldots, b f_{i}, f_{i+1}, \ldots, f_{m}\right)(x) \\
= & (b(x+h)-b(x)) T_{\epsilon}^{\vec{\alpha}}(\vec{f})(x+h) \\
& +\sum_{I \in \mathcal{D}} \epsilon_{I}\left(b(x)-b\left(x_{I}\right)\right) \prod_{j=1}^{m} \frac{\left\langle f_{j}, h_{I}^{1+\alpha_{j}}\right\rangle}{|I|} h_{I}^{\sigma(\vec{\alpha})}(x+h)
\end{aligned}
$$




$$
\begin{aligned}
& +\sum_{I \in \mathcal{D}} \epsilon_{I} \frac{\left\langle\left(b\left(x_{I}\right)-b\right) f_{i}, h_{I}^{1+\alpha_{i}}\right\rangle}{|I|} \prod_{1 \leq j \leq m, j \neq i} \frac{\left\langle f_{j}, h_{I}^{1+\alpha_{j}}\right\rangle}{|I|} h_{I}^{\sigma(\vec{\alpha})}(x+h) \\
& -\sum_{I \in \mathcal{D}} \epsilon_{I}\left(b(x)-b\left(x_{I}\right)\right) \prod_{j=1}^{m} \frac{\left\langle f_{j}, h_{I}^{1+\alpha_{j}}\right\rangle}{|I|} h_{I}^{\sigma(\vec{\alpha})}(x) \\
& +\sum_{I \in \mathcal{D}} \frac{\epsilon_{I}\left\langle\left(b\left(x_{I}\right)-b\right) f_{i}, h_{I}^{1+\alpha_{i}}\right\rangle}{|I|} \prod_{1 \leq j \leq m, j \neq i} \frac{\left\langle f_{j}, h_{I}^{1+\alpha_{j}}\right\rangle}{|I|} h_{I}^{\sigma(\vec{\alpha})}(x) \\
& =: \mathbb{I}_{1}+\mathbb{I}_{2}+\mathbb{I}_{3}-\mathbb{I}_{4}-\mathbb{I}_{5},
\end{aligned}
$$

where $x_{I}$ is the center of the dyadic interval $I$.

For $p$ with $1 / p=1 / p_{1}+\cdots+1 / p_{m}$, by the boundedness of $T_{\epsilon}^{\vec{\alpha}}$, we obtain

$$
\left\|\mathbb{I}_{1}\right\|_{p} \leq C\left\|b^{\prime}\right\|_{\infty}|h| \prod_{j=1}^{m}\left\|f_{j}\right\|_{p_{j}}
$$

Now, we estimate $\left\|\mathbb{I}_{2}-\mathbb{I}_{4}\right\|_{p}$. Similar as in the proof of Theorem 1.4 , we get

$$
\begin{aligned}
& \left(\int\left|\sum_{|I| \geq 1, I \cap(-1,1) \neq \emptyset} \epsilon_{I}\left(b(x)-b\left(x_{I}\right)\right) \prod_{j=1}^{m} \frac{\left\langle f_{j}, h_{I}^{1+\alpha_{j}}\right\rangle}{|I|}\left(h_{I}^{\sigma(\vec{\alpha})}(x+h)-h_{I}^{\sigma(\vec{\alpha})}(x)\right)\right|^{p} d x\right)^{1 / p} \\
\leq & \sum_{|I| \geq 1, I \cap(-1,1) \neq \emptyset}\left(\int\left|\epsilon_{I}\left(b(x)-b\left(x_{I}\right)\right) \prod_{j=1}^{m} \frac{\left\langle f_{j}, h_{I}^{1+\alpha_{j}}\right\rangle}{|I|}\left(h_{I}^{\sigma(\vec{\alpha})}(x+h)-h_{I}^{\sigma(\vec{\alpha})}(x)\right)\right|^{p} d x\right)^{1 / p} \\
\leq & C \sum_{|I| \geq 1, I \cap(-1,1) \neq \emptyset}\|b\|_{\infty}\left\|\left\{\epsilon_{I}\right\}\right\|_{\infty} \prod_{j=1}^{m}\left\|f_{j}\right\|_{p_{j}} \frac{|h|^{1 / p}}{|I|^{1 / p}} \leq C\|b\|_{\infty}\left\|\left\{\epsilon_{I}\right\}\right\|_{\infty} \prod_{j=1}^{m}\left\|f_{j}\right\|_{p_{j}}|h|^{1 / p} .
\end{aligned}
$$

If $|h|<|I|$, we take any $a<\min \{1,1 / p\}$ and have $|h|^{1 / p}|I|^{1-1 / p}<|h|^{a}|I|^{1-a}$. Then we obtain

$$
\begin{aligned}
& \left(\int\left|\epsilon_{I}\left(b(x)-b\left(x_{I}\right)\right) \prod_{j=1}^{m} \frac{\left\langle f_{j}, h_{I}^{1+\alpha_{j}}\right\rangle}{|I|}\left(h_{I}^{\sigma(\vec{\alpha})}(x+h)-h_{I}^{\sigma(\vec{\alpha})}(x)\right)\right|^{p} d x\right)^{1 / p} \\
\leq & \left\|b^{\prime}\right\|_{\infty}\left\|\left\{\epsilon_{I}\right\}\right\|_{\infty}|I| \prod_{j=1}^{m}\left|\frac{\left\langle f_{j}, h_{I}^{1+\alpha_{j}}\right\rangle}{|I|}\right|\left(\int\left|h_{I}^{\sigma(\vec{\alpha})}(x+h)-h_{I}^{\sigma(\vec{\alpha})}(x)\right|^{p} d x\right)^{1 / p} \\
\leq & C\left\|b^{\prime}\right\|_{\infty}\left\|\left\{\epsilon_{I}\right\}\right\|_{\infty} \prod_{j=1}^{m}\left\|f_{j} \chi_{I}\right\|_{p_{j}}|h|^{1 / p}|I|^{1-1 / p} \\
\leq & C\left\|b^{\prime}\right\|_{\infty}\left\|\left\{\epsilon_{I}\right\}\right\|_{\infty} \prod_{j=1}^{m}\left\|f_{j} \chi_{I}\right\|_{p_{j}}|h|^{a}|I|^{1-a} .
\end{aligned}
$$

When $|h| \geq|I|$, as in proof of Theorem 1.4, we have

$$
\left(\int\left|\epsilon_{I}\left(b(x)-b\left(x_{I}\right)\right) \prod_{j=1}^{m} \frac{\left\langle f_{j}, h_{I}^{1+\alpha_{j}}\right\rangle}{|I|}\left(h_{I}^{\sigma(\vec{\alpha})}(x+h)-h_{I}^{\sigma(\vec{\alpha})}(x)\right)\right|^{p} d x\right)^{1 / p}
$$




$$
\leq C\left\|b^{\prime}\right\|_{\infty}\left\|\left\{\epsilon_{I}\right\}\right\|_{\infty} \prod_{j=1}^{m}\left\|f_{j} \chi_{I}\right\|_{p_{j}}|I| \leq C\left\|b^{\prime}\right\|_{\infty}\left\|\left\{\epsilon_{I}\right\}\right\|_{\infty} \prod_{j=1}^{m}\left\|f_{j} \chi_{I}\right\|_{p_{j}}|h|^{a}|I|^{1-a} .
$$

Thus, for every $\ell \in \mathbb{N}$, it holds that

$$
\begin{aligned}
& \left(\int\left|\sum_{|I|=2^{-\ell}} \epsilon_{I}\left(b(x)-b\left(x_{I}\right)\right) \prod_{j=1}^{m} \frac{\left\langle f_{j}, h_{I}^{1+\alpha_{j}}\right\rangle}{|I|}\left(h_{I}^{\sigma(\vec{\alpha})}(x+h)-h_{I}^{\sigma(\vec{\alpha})}(x)\right)\right|^{p} d x\right)^{1 / p} \\
\leq & C\left\|b^{\prime}\right\|_{\infty}\left\|\left\{\epsilon_{I}\right\}\right\|_{\infty}|h|^{a} \sum_{|I|=2^{\ell}}|I|^{1-a} \prod_{j=1}^{m}\left\|f_{j} \chi_{I}\right\|_{p_{j}} \\
\leq & C\left\|b^{\prime}\right\|_{\infty}\left\|\left\{\epsilon_{I}\right\}\right\|_{\infty}|h|^{a} 2^{-(1-a) \ell} \prod_{j=1}^{m}\left\|f_{j}\right\|_{p_{j}} .
\end{aligned}
$$

This leads to

$$
\begin{aligned}
& \left(\int\left|\sum_{|I|<1, I \cap(-1,1) \neq \emptyset} \epsilon_{I}\left(b(x)-b\left(x_{I}\right)\right) \prod_{j=1}^{m} \frac{\left\langle f_{j}, h_{I}^{1+\alpha_{j}}\right\rangle}{|I|}\left(h_{I}^{\sigma(\vec{\alpha})}(x+h)-h_{I}^{\sigma(\vec{\alpha})}(x)\right)\right|^{p} d x\right)^{1 / p} \\
\leq & C\left\|b^{\prime}\right\|_{\infty}\left\|\left\{\epsilon_{I}\right\}\right\|_{\infty} \prod_{j=1}^{m}\left\|f_{j}\right\|_{p_{j}}|h|^{a} .
\end{aligned}
$$

Then we have $\left\|\mathbb{I}_{2}-\mathbb{I}_{4}\right\|_{p} \leq C\left\|b^{\prime}\right\|_{\infty}\left\|\left\{\epsilon_{I}\right\}\right\|_{\infty} \prod_{j=1}^{m}\left\|f_{j}\right\|_{p_{j}}|h|^{a}$. The estimate of $\left\|\mathbb{I}_{3}-\mathbb{I}_{5}\right\|_{p}$ is similar and we omit the details.

Therefore, we have shown that

$$
\lim _{h \rightarrow 0}\left\|\left[b, T_{\epsilon}^{\vec{\alpha}}\right]_{i}(\vec{f})(x+h)-\left[b, T_{\epsilon}^{\vec{\alpha}}\right]_{i}(\vec{f})(x)\right\|_{p}=0
$$

uniformly for $\vec{f}$ with $\left\|f_{j}\right\|_{p_{j}} \leq 1(j=1, \ldots, m)$.

(ii) Proof of compactness for iterated commutators. We will need the following lemma.

Lemma 5.3. Let $T$ be a multilinear operator and $b \in \mathrm{CMO}$. Suppose that $T$ is a compact operator from $L^{p_{1}}(\mathbb{R}) \times \cdots \times L^{p_{m}}(\mathbb{R})$ to $L^{p}(\mathbb{R})$. Then for any $i$ with $1 \leq i \leq m,[b, T]_{i}$ is a compact operator.

Proof. To illustrate $[b, T]_{i}$ is a compact operator, we only need to verify conditions (a), (b) and (c). Let $\mathcal{K}=\left\{[b, T]_{i}(\vec{f}):\|f\|_{p_{j}} \leq 1, j=1, \ldots, m\right\}$. We can deduce that $T$ is a bounded operator because $T$ is a compact operator. Therefore, we have

$$
\begin{aligned}
\left\|[b, T]_{i}(\vec{f})\right\|_{p} & \leq\|b\|_{\infty}\|T(\vec{f})\|_{p}+\left\|T\left(f_{1}, \ldots, b f_{i}, \ldots, f_{m}\right)\right\|_{p} \\
& \leq\left(\|b\|_{\infty} \prod_{j=1}^{m}\left\|f_{j}\right\|_{p_{j}}+\prod_{j \neq i}\left\|f_{j}\right\|_{p_{j}}\left\|b f_{i}\right\|_{p_{i}}\right) \\
& \leq 2\|b\|_{\infty} \prod_{j=1}^{m}\left\|f_{j}\right\|_{p_{j}} \leq 2\|b\|_{\infty},
\end{aligned}
$$


which implies that the condition (a) holds. Therefore, we only need to check that the conditions (b) and (c) hold. By the boundedness of $[b, T]_{i}(\vec{f})$, we may assume $b \in C_{c}^{\infty}(\mathbb{R})$. Due to the compactness of $T$, by the Fréchet-Kolmogorov-Riesz-Tsuji theorem, we have

$$
\begin{gathered}
\lim _{A \rightarrow \infty} \sup _{\left\|f_{j}\right\|_{p_{j}} \leq 1} \int_{|x| \geq A}|T(\vec{f})(x)|^{p} d x=0, \\
\lim _{h \rightarrow 0} \sup _{\left\|f_{j}\right\|_{p_{j}} \leq 1}\|T(\vec{f})(x+h)-T(\vec{f})(x)\|_{p}=0 .
\end{gathered}
$$

We assume that $M_{g}^{i}(\vec{f})=\left(f_{1}, \ldots, g f_{i}, \ldots, f_{m}\right)$. From (5.1), we see that

$$
\begin{aligned}
& \lim _{A \rightarrow \infty} \sup _{\left\|f_{j}\right\|_{p_{j}} \leq 1} \int_{|x| \geq A}\left|[b, T]_{i}(\vec{f})(x)\right|^{p} d x \\
\lesssim & \|b\|_{\infty}^{p} \lim _{A \rightarrow \infty} \sup _{\left\|f_{j}\right\|_{p_{j}} \leq 1}\left(\int_{|x| \geq A}|T(\vec{f})(x)|^{p} d x+\int_{|x| \geq A}\left|T\left(M_{\frac{b}{\|b\|_{\infty}}}^{i}(\vec{f})\right)(x)\right|^{p} d x\right)=0 .
\end{aligned}
$$

Obviously, it follows that

$$
\begin{aligned}
& \lim _{h \rightarrow 0} \sup _{\left\|f_{j}\right\|_{p_{j}} \leq 1}\left\|[b, T]_{i}(\vec{f})(x+h)-[b, T]_{i}(\vec{f})(x)\right\|_{p} \\
\leq & \lim _{h \rightarrow 0} \sup _{\left\|f_{j}\right\|_{p_{j}} \leq 1}\left(\|b(x+h) T(\vec{f})(x+h)-b(x) T(\vec{f})(x+h)\|_{p}\right. \\
& \left.+\|b(x) T(\vec{f})(x+h)-b(x) T(\vec{f})(x)\|_{p}+\left\|T\left(M_{b}^{i}(\vec{f})\right)(x+h)-T\left(M_{b}^{i}(\vec{f})\right)(x)\right\|_{p}\right) .
\end{aligned}
$$

By 5.2 and the boundedness of $T$, we deduce that

$$
\begin{aligned}
& \lim _{h \rightarrow 0} \sup _{\left\|f_{j}\right\|_{p_{j}} \leq 1}\left\|[b, T]_{i}(\vec{f})(x+h)-[b, T]_{i}(\vec{f})(x)\right\|_{p} \\
\leq & \left\|b^{\prime}\right\|_{\infty} \lim _{h \rightarrow 0} \sup _{\left\|f_{j}\right\|_{p_{j}} \leq 1}|h|\|T(\vec{f})(x+h)\|_{p}+\|b\|_{\infty} \lim _{h \rightarrow 0} \sup _{\left\|f_{j}\right\|_{p_{j}} \leq 1}\|T(\vec{f})(x+h)-T(\vec{f})(x)\|_{p} \\
& +\|b\|_{\infty} \lim _{h \rightarrow 0} \sup _{\left\|f_{j}\right\|_{p_{j}} \leq 1}\left\|T\left(M_{\frac{b}{\|b\|_{\infty}}}^{i}(\vec{f})\right)(x+h)-T\left(M_{\|b\|_{\infty}}^{i}(\vec{f})\right)(x)\right\|_{p}=0 .
\end{aligned}
$$

Therefore, $[b, T]_{i}$ is a compact operator for any $i$ with $1 \leq i \leq m$.

Now, by Lemma 5.3 and the compactness of commutator $\left[b, T_{\epsilon}^{\vec{\alpha}}\right]_{i}$, we can deduce that iterated commutators $T_{\epsilon, \Pi \mathbf{b}}^{\vec{\alpha}}$ is a compact operator for all $\vec{\alpha} \in\{0,1\}^{m} \backslash\{1, \ldots, 1\}$.

(iii) Proof of the compactness of $[b, \mathbb{S}]$. We need the following lemma for $[b, \mathbb{S}]$.

Lemma 5.4. Let $b \in \mathrm{BMO}\left(\mathbb{R}^{d}\right)$ and $1<p<\infty$. Then, for any $1<p<\infty$ there exists $C>0$ such that

$$
\|[b, \mathbb{S}](f)\|_{p} \leq C\|b\|_{\mathrm{BMO}}\|f\|_{p} .
$$


Proof. Let $x \in \mathbb{R}$ and $I_{0} \in \mathcal{D}$ contain $x$. Let $1<s<\infty$ and $I_{0}^{n}$ be the $n$-th ancestor of $I_{0}$. Then, it holds that

$$
[b, \mathbb{S}](f)(x)=\left(b(x)-b_{I_{0}}\right) \mathbb{S} f(x)-\mathbb{S}\left(\left(b-b_{I_{0}}\right) f \chi_{I_{0}^{n}}\right)(x)-\mathbb{S}\left(\left(b-b_{I_{0}}\right) f \chi_{\left(I_{0}^{n}\right)^{c}}\right)(x) .
$$

It is easy to show that

$$
\frac{1}{\left|I_{0}\right|} \int_{I_{0}}\left|\left(b(x)-b_{I_{0}}\right) \mathbb{S} f(z)\right| d z \leq C\|b\|_{\mathrm{BMO}} M_{s}^{d}(\mathbb{S}(f))(x)
$$

and

$$
\frac{1}{\left|I_{0}\right|} \int_{I_{0}}\left|\mathbb{S}\left(\left(b-b_{I_{0}}\right) f \chi_{I_{0}^{n}}\right)(z)\right| d z \leq C\|b\|_{\mathrm{BMO}} M_{s}^{d}(f)(x) .
$$

As for $\mathbb{S}\left(\left(b-b_{I_{0}}\right) f \chi_{\left(I_{0}^{n}\right)^{c}}\right)(x)$, take any $y \in I_{0}$ and denote $x_{I_{0}}$ to be the center of $I_{0}$. To estimate $\mathbb{S}\left(\left(b-b_{I_{0}}\right) f \chi_{\left(I_{0}^{n}\right)^{c}}\right)(x)$ on $I_{0}$, we only need to treat dyadic cubes $I, I^{\prime}$ and $I^{\prime \prime}$ satisfying $I^{\prime}, I^{\prime \prime} \subset I, \ell\left(I^{\prime}\right)=2^{-m} \ell(I), \ell\left(I^{\prime \prime}\right)=2^{-n} \ell(I), I^{\prime \prime} \cap I_{0} \neq \emptyset$ and $I^{\prime} \cap\left(I_{0}^{n}\right)^{c} \neq \emptyset$. If $I^{\prime \prime} \subset I_{0}$, it follows that $I_{0}^{n} \supset\left(I^{\prime \prime}\right)^{n}=I$, which contradicts $I^{\prime} \cap\left(I_{0}^{n}\right)^{c} \neq \emptyset$. So, we have $I_{0} \subsetneq I^{\prime \prime}$.

Since $h_{I^{\prime \prime}}$ is a constant on each child of $I^{\prime \prime}$, it is a constant on $I_{0}$. Hence we get

$$
\begin{aligned}
\mathbb{S}\left(\left(b-b_{I_{0}}\right) f \chi_{\left(I_{0}^{n}\right)^{c}}\right)(y) & =\sum_{\substack { I \in \mathcal{D} \\
\begin{subarray}{c}{l\left(I^{\prime}\right)=2^{-m} \in(I), l\left(I^{\prime \prime}\right)=2^{-n} l(I) \\
I^{\prime}, I^{\prime \prime} \in \mathcal{D}, I^{\prime}, I^{\prime \prime} \subset I{ I \in \mathcal { D } \\
\begin{subarray} { c } { l ( I ^ { \prime } ) = 2 ^ { - m } \in ( I ) , l ( I ^ { \prime \prime } ) = 2 ^ { - n } l ( I ) \\
I ^ { \prime } , I ^ { \prime \prime } \in \mathcal { D } , I ^ { \prime } , I ^ { \prime \prime } \subset I } }\end{subarray}} \frac{1}{|I|}\left\langle\left(b-b_{I_{0}}\right) \chi_{\left(I_{0}^{n}\right)^{c}} f, h_{I^{\prime}}\right\rangle h_{I^{\prime \prime}}(y) \\
& =\mathbb{S}\left(\left(b-b_{I_{0}}\right) f \chi_{\left(I_{0}^{n}\right)^{c}}\right)\left(x_{I_{0}}\right) .
\end{aligned}
$$

Thus we have

$$
M^{\#}([b, \mathbb{S}](f))(x) \leq C\|b\|_{\mathrm{BMO}}\left(M_{s}^{d}(\mathbb{S}(f))(x)+M_{s}^{d}(f)(x)\right)
$$

Let $b \in L^{\infty}\left(\mathbb{R}^{d}\right)$ and $f \in L^{p}\left(\mathbb{R}^{d}\right)$. Then, since $[b, \mathbb{S}](f)(x)=b(x) \mathbb{S}(f)(x)-\mathbb{S}(b f)(x)$, it follows that $[b, \mathbb{S}](f) \in L^{p}\left(\mathbb{R}^{d}\right)$. By inequality $(5.3)$, we see that

$$
\|[b, \mathbb{S}](f)\|_{p} \leq C\|b\|_{\mathrm{BMO}}\|f\|_{p} .
$$

Now, letting

$$
b_{j}(x)= \begin{cases}j & \text { if } b(x)>j \\ b(x) & \text { if }|b(x)| \leq j \\ -j & \text { if } b(x)<-j\end{cases}
$$

and taking a subsequence (if necessary), we can deduce that (cf. [11]):

$$
\|[b, \mathbb{S}](f)\|_{p} \leq C\|b\|_{\mathrm{BMO}}\|f\|_{p}
$$


Now we turn to the proof of (iii). We may assume $b \in C_{c}^{\infty}\left(\mathbb{R}^{d}\right)$ with $\operatorname{supp} b \subset(-1,1)^{d}$. For the sake of simplicity, we only consider the integration on $E_{k}:=[0, \infty)^{d} \backslash\left[0,2^{k}\right)^{d}$. Notice that

$$
\begin{aligned}
& \int_{E_{k}}|[b, \mathbb{S}](f)(x)|^{p} d x \\
& =\int_{E_{k}}\left|\sum_{I=\left[0,2^{\ell}\right)^{d}, \ell \geq k} \sum_{\substack{I^{\prime}, I^{\prime \prime} \in \mathcal{D}, I^{\prime}, I^{\prime \prime} \subset I \\
l\left(I^{\prime}\right)=2^{-m} l(I), l\left(I^{\prime \prime}\right)=2^{-n} l(I)}} \frac{1}{|I|}\left\langle b f, h_{I^{\prime}}\right\rangle h_{I^{\prime \prime}}(x)\right|^{p} d x \\
& \leq C \int_{E_{k}}\left(\sum_{I=\left[0,2^{\ell}\right)^{d}, \ell \geq k} \sum_{\substack{I^{\prime}, I^{\prime \prime} \in \mathcal{D}, I^{\prime}, I^{\prime \prime} \subset I \\
l\left(I^{\prime}\right)=2^{-m} l(I), l\left(I^{\prime \prime}\right)=2^{-n} l(I)}} \frac{\|b\|_{\infty}\left\|f \chi_{[0,1)^{d}}\right\|_{1}\left\|h_{I^{\prime}}\right\|_{\infty}\left\|h_{I^{\prime \prime}}\right\|_{\infty}}{|I|} \chi_{I^{\prime \prime}}(x)\right)^{p} d x \\
& \leq C\|b\|_{\infty}^{p}\|f\|_{p}^{p} \int_{E_{k}}\left(\sum_{I=\left[0,2^{\ell}\right)^{d}, \ell \geq k}|I|^{-1} \chi_{I}(x)\right)^{p} d x \leq C\|b\|_{\infty}^{p}\|f\|_{p}^{p} \times 2^{-(p-1) d k},
\end{aligned}
$$

which yields that

$$
\lim _{A \rightarrow \infty} \int_{|x| \geq A}|[b, \mathbb{S}](f)(x)|^{p} d x=0
$$

uniformly for $f$ with $\|f\|_{p} \leq 1$. This shows that condition (b) holds.

Now, we are ready to check condition $(c)$. We rewrite $[b, \mathbb{S}](f)(x+h)-[b, \mathbb{S}](f)(x)$ in the following way

$$
\begin{aligned}
& {[b, \mathbb{S}](f)(x+h)-[b, \mathbb{S}](f)(x)} \\
& =(b(x+h)-b(x)) \mathbb{S}(f)(x+h)
\end{aligned}
$$

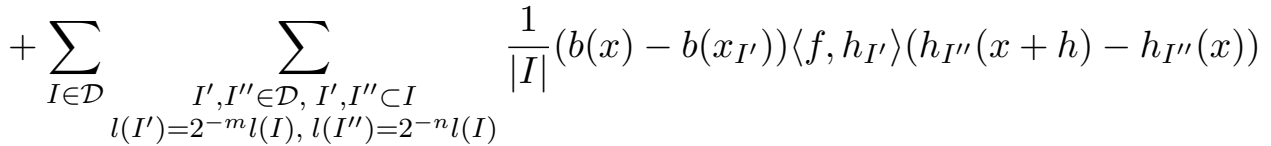

$$
\begin{aligned}
& -\sum_{I \in \mathcal{D}} \sum_{\substack{I^{\prime}, I^{\prime \prime} \in \mathcal{D}, I^{\prime}, I^{\prime \prime} \subset I \\
l\left(I^{\prime}\right)=2^{-m} l(I), l\left(I^{\prime \prime}\right)=2^{-n} l(I)}} \frac{1}{|I|}\left\langle\left(b-b\left(x_{I^{\prime}}\right)\right) f, h_{I^{\prime}}\right\rangle\left(h_{I^{\prime \prime}}(x+h)-h_{I^{\prime \prime}}(x)\right) \\
& =: I I_{1}+I I_{2}+I I_{3} \text {. }
\end{aligned}
$$

The $L^{p}$ boundedness of $\mathbb{S}$ yields that

$$
\left\|I I_{1}\right\|_{p} \leq C\|\nabla b\|_{\infty}\|f\|_{p}|h| .
$$

For $I, I^{\prime}, I^{\prime \prime} \in \mathcal{D}$ satisfying $|I| \geq 1, I \cap(-1,1)^{d} \neq \emptyset$, and $I^{\prime}, I^{\prime \prime} \subset I, l\left(I^{\prime}\right)=2^{-m} l(I)$, $l\left(I^{\prime \prime}\right)=2^{-n} l(I)$, we have

$$
\begin{aligned}
& \left(\int\left|\frac{1}{|I|}\left(b(x)-b\left(x_{I^{\prime}}\right)\right)\left\langle f, h_{I^{\prime}}\right\rangle\left(h_{I^{\prime \prime}}(x+h)-h_{I^{\prime \prime}}(x)\right)\right|^{p} d x\right)^{1 / p} \\
\leq & C\|b\|_{\infty}|I|^{-1}\left\|f \chi_{I^{\prime}}\right\|_{p}\left|I^{\prime}\right|^{1 / p^{\prime}}\left\|h_{I^{\prime}}\right\|_{\infty}\left\|h_{I^{\prime \prime}}\right\|_{\infty}\left(|h||I|^{(d-1) / d}\right)^{1 / p} \\
\leq & C\|b\|_{\infty}\|f\|_{p}|h|^{1 / p}|I|^{-1 /(d p)} .
\end{aligned}
$$


Thus, noting that $\operatorname{supp} b \subset(-1,1)^{d}$, we get

$$
\begin{gathered}
\left(\int \mid \sum_{\substack{I \in \mathcal{D},|I| \geq 1, I \cap(-1,1)^{d} \neq \emptyset l\left(I^{\prime}\right)=2^{-m} l(I), l\left(I^{\prime \prime}\right)=2^{-n} l(I)}} \sum_{\substack{I^{\prime}, I^{\prime \prime} \in \mathcal{D}, I^{\prime}, I^{\prime \prime} \subset I \\
|I|}} \frac{1}{\left.\mid b(x)-b\left(x_{I^{\prime}}\right)\right)\left\langle f, h_{I^{\prime}}\right\rangle}\right. \\
\left.\times\left.\left(h_{I^{\prime \prime}}(x+h)-h_{I^{\prime \prime}}(x)\right)\right|^{p} d x\right)^{1 / p} \\
\leq C\|b\|_{\infty}\|f\|_{p}|h|^{1 / p} \cdot 2^{(m+n) d} \sum_{I=\left[0,2^{\ell}\right)^{d}, \ell \in \mathbb{N}}|I|^{-1 /(d p)} \leq C\|b\|_{\infty}\|f\|_{p}|h|^{1 / p} .
\end{gathered}
$$

Next, we treat the case $|h|^{d}<|I|<1$. For every $\ell \in \mathbb{N}$, using the support of $h_{I}^{\sigma(\vec{\alpha})}(x+$ $h)-h_{I}^{\sigma(\vec{\alpha})}(x)$ and by Hölder's inequality, we get

$$
\begin{aligned}
& \left(\int \mid \sum_{\substack{I \in \mathcal{D},|h|^{d}<|I|=2^{-d \ell} \\
I \cap(-1,1)^{d} \neq \emptyset}} \sum_{\substack{I^{\prime}, I^{\prime \prime} \in \mathcal{D}, I^{\prime}, I^{\prime \prime} \subset I \\
l\left(I^{\prime}\right)=2^{-m} l(I), l\left(I^{\prime \prime}\right)=2^{-n} l(I)}} \frac{1}{|I|}\left(b(x)-b\left(x_{I^{\prime}}\right)\right)\right. \\
& \left.\times\left.\left\langle f, h_{I^{\prime}}\right\rangle\left(h_{I^{\prime \prime}}(x+h)-h_{I^{\prime \prime}}(x)\right)\right|^{p} d x\right)^{1 / p} \\
& \leq C\|\nabla b\|_{\infty}\left(\left.\left.\int\left|\sum_{\substack{I \in \mathcal{D},|h|^{d}<|I|=2^{-d \ell} \\
I \cap(-1,1)^{d} \neq \emptyset}} \sum_{\substack{I^{\prime \prime} \in \mathcal{D}, I^{\prime \prime} \subset I \\
l\left(I^{\prime \prime}\right)=2^{-n} l(I)}}\right| I\right|^{\frac{1}{d}-\frac{1}{p}}\left\|f \chi_{I}\right\|_{p} \frac{\left|h_{I^{\prime \prime}}(x+h)-h_{I^{\prime \prime}}(x)\right|}{\left\|h_{I^{\prime \prime}}\right\|_{\infty}}\right|^{p} d x\right)^{1 / p} \\
& \leq C\|\nabla b\|_{\infty}\left(\sum_{\substack{I \in \mathcal{D},|h|^{d}<|I|=2^{-d \ell} \\
I \cap(-1,1)^{d} \neq \emptyset}}|I|^{\frac{p}{d}-1}\left\|f \chi_{I}\right\|_{p}^{p}|h||I|^{\frac{d-1}{d}}\right)^{1 / p} \\
& \leq C 2^{-\ell(1-1 / p)}\|\nabla b\|_{\infty}\|f\|_{p}|h|^{1 / p} \text {. }
\end{aligned}
$$

Thirdly, noting that $|I|^{1 / d}<|h|$ implies $|h| /|I|^{1 / d}>1$, we get

$$
\begin{aligned}
& \left(\int \sum_{\substack{I \in \mathcal{D},|I|=2^{-d \ell} \leq|h|^{d}<1, I \cap(-1,1)^{d} \neq \emptyset}} \sum_{\substack{I^{\prime}, I^{\prime \prime} \in \mathcal{D}, I^{\prime}, I^{\prime \prime} \subset I \\
l\left(I^{\prime}\right)=2^{-m} l(I), l\left(I^{\prime \prime}\right)=2^{-n} l(I)}} \frac{1}{|I|}\left(b(x)-b\left(x_{I^{\prime}}\right)\right)\right. \\
& \left.\times\left.\left\langle f, h_{I^{\prime}}\right\rangle\left(h_{I^{\prime \prime}}(x+h)-h_{I^{\prime \prime}}(x)\right)\right|^{p} d x\right)^{1 / p} \\
& \left.\leq C\|\nabla b\|_{\infty}\left(\sum_{\substack{I \in \mathcal{D},|I|=2^{-d \ell} \leq|h|^{d}<1, I \cap(-1,1)^{d} \neq \emptyset}}|I|^{\frac{p}{d}} \frac{|h|}{|I|^{\frac{1}{d}}}\left\|f \chi_{I}\right\|_{p}^{p}\right)^{1 / p}\left\|f \chi_{I}\right\|_{p}^{p}\right)^{1 / p} \\
& \leq C 2^{-\ell(1-1 / p)}\|\nabla b\|_{\infty}|h|^{1 / p}\left(\sum_{\substack{I \in \mathcal{D},|I|=2^{d \ell} \leq|h|^{d}<1, I \cap(-1,1)^{d} \neq \emptyset}}\right. \\
& \leq C 2^{-\ell(1-1 / p)}\|\nabla b\|_{\infty}\|f\|_{p}|h|^{1 / p} .
\end{aligned}
$$


Hence

$$
\begin{aligned}
& \left(\int \mid \sum_{\substack{I \in \mathcal{D},|I|<1, I \cap(-1,1)^{d} \neq \emptyset}} \sum_{\substack{\left.\left.I^{\prime}, I^{\prime \prime} \in \mathcal{D}, I^{\prime}\right)=2^{-m} l(I), I^{\prime \prime} \subset I \\
I^{\prime \prime}\right)=2^{-n} l(I)}} \frac{1}{|I|}\left(b(x)-b\left(x_{I^{\prime}}\right)\right)\right. \\
& \left.\quad \times\left.\left\langle f, h_{I^{\prime}}\right\rangle\left(h_{I^{\prime \prime}}(x+h)-h_{I^{\prime \prime}}(x)\right)\right|^{p} d x\right)^{1 / p} \\
& \leq \sum_{\ell=1}^{\infty} C 2^{-\ell(1-1 / p)}\|\nabla b\|_{\infty}\|f\|_{p}|h|^{1 / p} \\
& \leq C\|\nabla b\|_{\infty}\|f\|_{p}|h|^{1 / p} .
\end{aligned}
$$

Thus, we obtain that $\left\|I I_{2}\right\|_{p} \leq C\|\nabla b\|_{\infty}\|f\|_{p}|h|^{1 / p}$. Similarly we have the same estimate for $\left\|I I_{3}\right\|_{p}$.

Therefore, we have shown that $[b, \mathbb{S}]$ satisfies conditions $(\mathrm{a})-(\mathrm{c})$ and $[b, \mathbb{S}]$ is a compact operator.

\section{Appendix}

In this section, we shall give a lemma, which was used in the proof of Theorem 1.4 .

Lemma 6.1. Let $X_{1}, \ldots, X_{m}$ be Banach spaces with norms $\|\cdot\|_{X_{j}}(j=1, \ldots, m)$ and $Y$ be a complete quasi-normed vector space with quasi-norm $\|\cdot\|_{Y}$. For every $n \in \mathbb{N}$ let $T_{n}$ be a bounded m-linear operator from $X_{1} \times \cdots \times X_{m}$ to $Y$, which is a compact operator, and $T$ be an $m$-linear operator from $X_{1} \times \cdots \times X_{m}$ to $Y$ satisfying

$$
\lim _{n \rightarrow \infty} \sup _{0 \neq \vec{f} \in X_{1} \times \cdots \times X_{m}} \frac{\left\|T_{n}(\vec{f})-T(\vec{f})\right\|_{Y}}{\prod_{\ell=1}^{m}\left\|f_{\ell}\right\|_{X_{\ell}}}=0
$$

where $\vec{f}=\left(f_{1}, \ldots, f_{m}\right)$. Then $T$ is also a compact operator from $X_{1} \times \cdots \times X_{m}$ to $Y$.

Proof. We borrow the proof idea from Yosida's book [20, p. 278]. Let $\left\{\vec{g}_{j}\right\}_{j=1}^{\infty}$ be a bounded sequence in $X_{1} \times \cdots \times X_{m}$ such $\left\|g_{j, l}\right\|_{X_{l}} \leq 1(l=1, \ldots, m)$, where $\vec{g}_{j}=\left(g_{j, 1}, \ldots, g_{j, m}\right)$. By the compact property of each $T_{n}$, we can choose, by the diagonal method, a subsequence $\left\{\vec{f}_{j}\right\}_{j=1}^{\infty}$ of $\left\{\vec{g}_{j}\right\}_{j=1}^{\infty}$ such that $\lim _{j \rightarrow \infty} T_{n}\left(\vec{f}_{j}\right)$ exists in $Y$ for every fixed $n \in \mathbb{N}$. Noting that $\left\|f_{j, \ell}\right\|_{X_{\ell}} \leq 1(l=1, \ldots, m)$ implies $\prod_{\ell=1}^{m}\left\|f_{j, \ell}\right\|_{X_{\ell}} \leq 1$, we have for every $n$,

$$
\begin{aligned}
\left\|T\left(\vec{f}_{j}\right)-T\left(\overrightarrow{f_{l}}\right)\right\|_{Y} & \leq C\left(\left\|T\left(\vec{f}_{j}\right)-T_{n}\left(\vec{f}_{j}\right)\right\|_{Y}+\left\|T_{n}\left(\vec{f}_{j}\right)-T_{n}\left(\overrightarrow{f_{l}}\right)\right\|_{Y}+\left\|T_{n}\left(\overrightarrow{f_{l}}\right)-T\left(\overrightarrow{f_{l}}\right)\right\|_{Y}\right) \\
& \leq 2 C \sup _{0 \neq \vec{f} \in X_{1} \times \cdots \times X_{m}} \frac{\left\|T_{n}(\vec{f})-T(\vec{f})\right\|_{Y}}{\prod_{\ell=1}^{m}\left\|f_{\ell}\right\|_{X_{\ell}}}+C\left\|T_{n}\left(\vec{f}_{j}\right)-T_{n}\left(\overrightarrow{f_{l}}\right)\right\|_{Y} .
\end{aligned}
$$

Hence

$$
\varlimsup_{j, l \rightarrow \infty}\left\|T\left(\overrightarrow{f_{j}}\right)-T\left(\overrightarrow{f_{l}}\right)\right\|_{Y} \leq 2 C \sup _{0 \neq \vec{f} \in X_{1} \times \cdots \times X_{m}} \frac{\left\|T_{n}(\vec{f})-T(\vec{f})\right\|_{Y}}{\prod_{\ell=1}^{m}\left\|f_{\ell}\right\|_{X_{\ell}}}
$$


and so $\left\{T\left(\vec{f}_{j}\right)\right\}_{j=1}^{\infty}$ is a Cauchy sequence in $Y$. This shows that $T$ is a compact operator from $X_{1} \times \cdots \times X_{m}$ to $Y$.

\section{Acknowledgments}

The authors want to express their sincere thanks to the referee for his or her valuable remarks and suggestions, which made this paper more readable.

\section{References}

[1] Á. Bényi and R. H. Torres, Compact bilinear operators and commutators, Proc. Amer. Math. Soc. 141 (2013), no. 10, 3609-3621.

[2] J.-M. Bony, Calcul symbolique et propagation des singularités pour les équations aux dérivées partielles non linéaires, Ann. Sci. École Norm. Sup. (4) 14 (1989), no. 2, 209-246.

[3] G. David and J.-L. Journé, A boundedness criterion for generalized CalderónZygmund operators, Ann. of Math. (2) 120 (1984), no. 2, 371-397.

[4] Y. Ding, T. Mei and Q. Xue, Compactness of maximal commutators of bilinear Calderón-Zygmund singular integral operators, in: Some Topics in Harmonic Analysis and Applications, 163-175, Adv. Lect. Math. (ALM) 34, Int. Press, Somerville, MA, 2016.

[5] R. Hanks, Interpolation by the real method between $\mathrm{BMO}, L^{\alpha}(0<\alpha<\infty)$ and $H^{\alpha}$ $(0<\alpha<\infty)$, Indiana Univ. Math. J. 26 (1977), no. 4, 679-689.

[6] T. Hytönen, C. Pérez, S. Treil and A. Volberg, Sharp weighted estimates for dyadic shifts and the $A_{2}$ conjecture, J. Reine Angew. Math. 687 (2014), 43-86.

[7] F. John and L. Nirenberg, On functions of bounded mean oscillation, Comm. Pure Appl. Math. 14 (1961), 415-426.

[8] I. Kunwar, Multilinear dyadic operators and their commutators in the weighted setting, arXiv:1512.04630.

[9] - Multilinear dyadic operators and their commutators, Ann. Univ. Ferrara Sez. VII Sci. Mat. 64 (2018), no. 1, 111-144.

[10] M. T. Lacey, S. Petermichl and M. C. Reguera, Sharp $A_{2}$ inequality for Haar shift operators, Math. Ann. 348 (2010), no. 1, 127-141. 
[11] A. K. Lerner, S. Ombrosi, C. Pérez, R. H. Torres and R. Trujillo-González, New maximal functions and multiple weights for the multilinear Calderón-Zygmund theory, Adv. Math. 220 (2009), no. 4, 1222-1264.

[12] F. Nazarov, S. Treil and A. Volberg, Two weight inequalities for individual Haar multipliers and other well localized operators, Math. Res. Lett. 15 (2008), no. 3, 583597.

[13] C. Pérez, G. Pradolini, R. H. Torres and R. Trujillo-González, End-point estimates for iterated commutators of multilinear singular integrals, Bull. Lond. Math. Soc. 46 (2014), no. 1, 26-42.

[14] S. Petermichl and A. Volberg, Heating of the Ahlfors-Beurling operator: weakly quasiregular maps on the plane are quasiregular, Duke Math. J. 112 (2002), no. 2, 281-305.

[15] M. Tsuji, On the compactness of space $L^{p}(p>0)$ and its application to integral equations, Kodai Math. Sem. Rep. 3 (1951), 33-36.

[16] A. Uchiyama, On the compactness of operators of Hankel type, Tohoku Math. J. (2) 30 (1978), no. 1, 163-171.

[17] S. Wang, The compactness of the commutator of fractional integral operator, Chin. Ann. Math. 8(A) (1987), 475-482.

[18] J. Wittwer, A sharp estimate on the norm of the martingale transform, Math. Res. Lett. 7 (2000), no. 1, 1-12.

[19] Q. Xue, Weighted estimates for the iterated commutators of multilinear maximal and fractional type operators, Studia Math. 217 (2011), no. 2, 97-122.

[20] K. Yosida, Functional Analysis, Classics in Mathematics, Springer-Verlag, Berlin, 1995.

Heng Gu and Qingying Xue

School of Mathematical Sciences, Beijing Normal University, Laboratory of Mathematics and Complex Systems, Ministry of Education, Beijing 100875, China

E-mail addresses: henggu@mail.bnu.edu.cn, qyxue@bnu.edu.cn

Kôzô Yabuta

Research Center for Mathematical Sciences, Kwansei Gakuin University, Gakuen 2-1, Sanda 669-1337, Japan

E-mail address: kyabuta3@kwansei.ac.jp 